\title{
Nanotheranostics
}

2017; 1(1): 1-22. doi: 10.7150/ntno.17109

Review

\section{Remotely Triggered Nano-Theranostics For Cancer Applications}

\author{
Alexandra Sneider, Derek VanDyke, Shailee Paliwal, Prakash Rai ${ }^{\bowtie}$ \\ University of Massachusetts Lowell, Department of Chemical Engineering, Francis College of Engineering, 1 University Ave, Lowell, MA 01854, USA. \\ $\bowtie$ Corresponding author: Prakash Rai, Phone 978-934-4971, prakash_rai@uml.edu. \\ (C) Ivyspring International Publisher. This is an open access article distributed under the terms of the Creative Commons Attribution (CC BY-NC) license \\ (https://creativecommons.org/licenses/by-nc/4.0/). See http://ivyspring.com/terms for full terms and conditions.
}

Received: 2016.08.04; Accepted: 2016.10.16; Published: 2017.01.01

\begin{abstract}
Nanotechnology has enabled the development of smart theranostic platforms that can concurrently diagnose disease, start primary treatment, monitor response, and, if required, initiate secondary treatments. Recent in vivo experiments demonstrate the promise of using theranostics in the clinic. In this paper, we review the use of remotely triggered theranostic nanoparticles for cancer applications, focusing heavily on advances in the past five years. Remote triggering mechanisms covered include photodynamic, photothermal, phototriggered chemotherapeutic release, ultrasound, electro-thermal, magneto-thermal, X-ray, and radiofrequency therapies. Each section includes a brief overview of the triggering mechanism and summarizes the variety of nanoparticles employed in each method. Emphasis in each category is placed on nano-theranostics with in vivo success. Some of the nanotheranostic platforms highlighted include photoactivatable multi-inhibitor nanoliposomes, plasmonic nanobubbles, reduced graphene oxide-iron oxide nanoparticles, photoswitching nanoparticles, multispectral optoacoustic tomography using indocyanine green, low temperature sensitive liposomes, and receptor-targeted iron oxide nanoparticles loaded with gemcitabine. The studies reviewed here provide strong evidence that the field of nanotheranostics is rapidly evolving. Such nanoplatforms may soon enable unique advances in the clinical management of cancer. However, reproducibility in the synthesis procedures of such "smart" platforms that lend themselves to easy scale-up in their manufacturing, as well as the development of new and improved models of cancer that are more predictive of human responses, need to happen soon for this field to make a rapid clinical impact.
\end{abstract}

Key words: nanomedicine, oncology, externally-triggered, image guided, photoactivated.

\section{Introduction}

The incidence and mortality predictions for cancer in 2016 include 1,685,210 new cases, and an estimated 595,690 deaths within the United States alone [1]. While there have been improvements to these statistics as a result of targeted treatments, early detection, and behavioral changes, many treatment options continue to be ineffective at preventing recurrence. Additionally, these treatment options often involve invasive procedures, drug resistance, and systemic toxicity [1]. Successful, non-invasive delivery of biodegradable and non-toxic combination therapies that can target existing cancerous cells and prevent the development of new tumors all in one dose is ideal for clinical treatment. These novel technologies that can intelligently and effectively remove the diseased tissue with limited negative effect to the patient, and minimize the chance of cancer recurrence, could make an immediate impact in the clinic [2-9]. When designing these technologies, it is important to focus on adequate circulation time, specific delivery to cancerous tissue only, evasion of normal tissues and accumulation in the organs, lack of an immune response, and concomitant treatment and noninvasive monitoring in order for successful drug delivery to occur. The use of remotely triggered treatments in combination with imaging modalities 
such as ultrasound, computed tomography, magnetic resonance imaging, and positron emission tomography could prove to address many of the current issues in cancer treatment, possibly leading to significant clinical outcomes [10-12].

In 2002, Funkhouser coined the term "theranostics" to represent a new class of treatment options that are capable of providing both therapy and diagnostics to patients [13]. The eventual goal of these therapies is to provide targeted, individualized treatment options that allow health professionals to monitor the cancer tissue, trigger release of therapeutics, and monitor the response to the release. Theranostics would lead to better survival statistics and quality of life by causing enhanced treatment at the cancer sites while reducing toxic effects. Pioneering work that showcases the beneficial effects of theranostics to cancer treatment is best characterized by Lukianova-Hleb and colleagues' work with plasmonic nanobubbles [14,15], highlighted later in this article [16-18]. This review will focus specifically on remotely-triggered treatments in combination with an imaging modality, providing a theranostic platform.

"Smart" systems that release drugs or induce a toxic effect in response to an externally controlled stimulus promise unique clinical benefits over conventional systems that release their cargo passively or are activated internally. These systems are referred to as remotely triggered systems. Externally controlled triggers include visible or near-infrared (NIR) light, X-rays, radiofrequency waves, ultrasound, and electric or magnetic fields. Multifunctional nanoparticle-based remotely triggered theranostics that permit simultaneous cancer diagnosis and therapy have been designed, synthesized, and evaluated in various in vitro and in vivo models of cancer [19-34]. It is also important to note that, if required, multiple treatment modalities can be integrated into a single nanostructure for multimodal imaging and therapy. These multimodal theranostics afford the possibility of eliminating cancer cells using a combination of two or more remotely triggered therapies (e.g. phototherapy, chemotherapy, radiotherapy, etc.). New treatment options that minimize damage to otherwise healthy living tissue through non-invasive remotely triggered treatments would be desirable to reduce morbidity in patients. Additionally, it would be helpful if these remotely triggered treatments could be augmented to have diagnostic and treatment monitoring abilities, leading to a remotely triggered theranostic platform that could prove to be of clinical significance.

Remotely triggered theranostics could allow for early recognition of the disease and the controlled, image-guided release of a therapeutic agent [35]. By using contrast agents and imaging techniques (MRI, optical imaging, ultrasound, X-ray) the location of the disease can be targeted using these remote triggers, allowing for control of the location of drug release and subsequent cell death. With more information about the tumor, there is a reduced potential for over and under dosing that allows for better control over the quantity of drug released. In addition, remotely triggered therapies can provide control over when the treatment occurs and the treatment duration, which is not possible with most conventional approaches. Through the use of remotely triggered theranostics, treatments can either be turned "on" or "off" depending on a variety of factors, including whether or not the nanoparticle has reached the tumor sites. This ability to turn treatments "on" or "off," compared to a treatment always being "on" as in previous treatment methods, allows for reduced toxicity in noncancerous or "normal" tissue. This control over location, quantity, and timing allows for tailored drug release profiles and localized treatments that reduce toxicity to surrounding tissue. Not only are remotely activated treatments beneficial for triggered chemotherapeutic release, but they are also advantageous regardless of the type of treatment due to their non-invasive nature, which is desirable for clinical translation and patient compliance [36]. The non-invasive nature is a characteristic of many remote-triggering systems (NIR light, electric/magnetic fields, and ultrasound) that possess the ability to penetrate through skin, and in some cases through tissue, to induce a treatment (i.e. thermal or chemotherapeutic release).

One such example of a clinical application of a remotely triggered treatment is ThermoDox, which is in Phase III clinical trials. This novel liposomal drug delivery system is a temperature sensitive system that releases doxorubicin from its core when there is a certain increase in temperature produced by remote triggers like a radiofrequency (RF) pulse or high intensity focused ultrasound (HIFU). Additionally, ThermoDox could be combined with an imaging modality to provide a remotely triggered theranostic effect. This is just one example of the success remotely triggered treatments have achieved as a result of their unique treatment aspects [37].

The objective of this review is to discuss some major advances in the field of remotely triggered nano-theranostics for cancer applications by briefly summarizing the development and clinical potential of various remotely triggered theranostics (photodynamic, photothermal, phototriggered chemotherapeutic release, ultrasound, electrothermal, magneto-thermal, $\mathrm{X}$-ray, and radiofre- 
quency) and delineating the challenges that must be overcome for successful clinical development and implementation of such cancer theranostics. Each stimulus section includes at least one highlighted article focusing on in vivo application of that nano-theranostic and acknowledges additional applications of the therapy.

\section{Phototriggered Theranostics for Cancer Applications}

Light-sensitive multifunctional nanoparticles can be used to locate cancer in a patient using various imaging modalities, including optical imaging. Using an external light source as a trigger, these nanoparticles can then be used for targeted and on call drug release at the cancer sites. Such photo-triggered theranostics will result in better treatments that eradicate the possibility of under or overdosing, reduce the requirement for multiple rounds of administrations, and lead to improved patient compliance. This section will focus on photodynamic therapy, photothermal therapy, and phototriggered chemotherapeutic release. Currently, photodynamic therapy is clinically approved for cancer treatment, and photothermal therapy and phototriggered chemotherapeutic release are still in clinical trials for cancer treatment [36].

\section{Photodynamic Theranostics}

Photodynamic therapy (PDT) involves the use of a non-toxic agent, known as a photosensitizer (PS), that is irradiated with light to induce the formation of reactive oxygen species (ROS) that stimulate cellular destruction at the region of interest [38]. Nanoparticle delivery of the PS agents overcomes delivery limitations due to PS hydrophobicity, non-specific targeting, and toxicity. Targeting of various cancers using this method depends on the ability of the laser. There must be control of the wavelength, fluence, and irradiation time in order to penetrate tissue and reach the PS. For example, head, neck, ocular, and breast cancers may be treated with an external laser source, while intestinal or pancreatic cancers may be reached using a laser on the end of an endoscope.

Spring et al., in their 2016 Nature Nanotechnology article, used already FDA approved materials to develop a phototriggered multi-inhibitor nanoliposome (PMIL) encapsulating the photosensitive, non-cytotoxic, hydrophobic drug benzoporphyrin derivative (BPD) within the lipid bilayer, and PLGA-PEG conjugated cabozantinib (NP[XL184]) inside to target human pancreatic ductal adenocarcinoma (PDAC) (150 nm, PDI 0.14, $-3 \mathrm{mV}$, $50 \%$ BPD release after 312 hours) [39]. Irradiation of the PMIL via $690 \mathrm{~nm}$ near-infrared light triggers BPD leading to PDT induced tumor cell apoptosis and microvessel damage and simultaneous release of NP[XL184] to inhibit anti-apoptotic, vascular endothelial growth factor (VEGF), and the tyrosine kinase for hepatocyte growth factor (MET) receptor signaling. A depiction of this treatment mechanism can be seen in Figure 1A. In vivo administration and triggering of a single dose of PMIL in subcutaneous AsPC1 (Figure 1B) and orthotopic PDAC (Figure 1C) metastatic mouse models showed $92 \%$ and $61 \%$ respective reductions in tumor volume, $70 \%$ reduction in intratumoral microvessel volume (Figure 1D), and 99\% reduction of metastatic cancer cells traveling to the liver and regional lymph nodes (Figure 1E). These outcomes are comparable to the daily oral administration of systemically toxic, unencapsulated XL184 for three weeks in mouse models of pancreatic neuroendocrine tumors. These results show promise for future clinical treatment in humans. Next steps include specific targeting agents for tumor cells and micrometastases, and optimizing encapsulation of $\mathrm{NP}[\mathrm{XL184}]$ to further reduce the small molecule toxicity. [39]

There are other promising photodynamic therapies for the treatment of cancer. Hsp90 loaded porphyrin based telodendrimers were used to image drug delivery and treat tumors in prostate cancer in mice through the variable release of Hsp90 and localized irradiation, limiting cytotoxicity [40]. Additionally, $\mathrm{NaYF}_{4}: \mathrm{Yb}^{3+}, \mathrm{Er}^{3+}$ upconversion nanoparticles with the photosensitive molecule Rose Bengal and folic acid targeting agent, which is triggered at $980 \mathrm{~nm}$, shows promise for both the imaging and treatment of JAR choriocarcinoma in vivo [29]. The same PS was also used with silica nanoparticles to treat breast and oral cancer cell lines in vitro [41]. Additionally, folic acid functionalized carbon nanodots carrying zinc pthalocyanine PS were used to target, image, and treat human cervical cancer HeLa cells [43].

Other nanoparticles used for PDT include upconverted nanoparticles [26, 27, 28, 29, 42-47], hyaluronic acid derivatized carbon nanotubes [48], selenium-rubyrin loaded nanoparticles [49], gold vesicles [19], silica coated titanium dioxide [20], cobalt ferrite nanoparticles [21], lipid calcium phosphate nanoparticles [22], small molecule quenched activity-based probes [23], carbon dots [24, 30], polyacrylamide nanoparticles [25], calcium phosphosilicate nanoparticles [50], mesoporous silicon nanoparticles [51, 52], and gold nanoparticles [53-55]. 

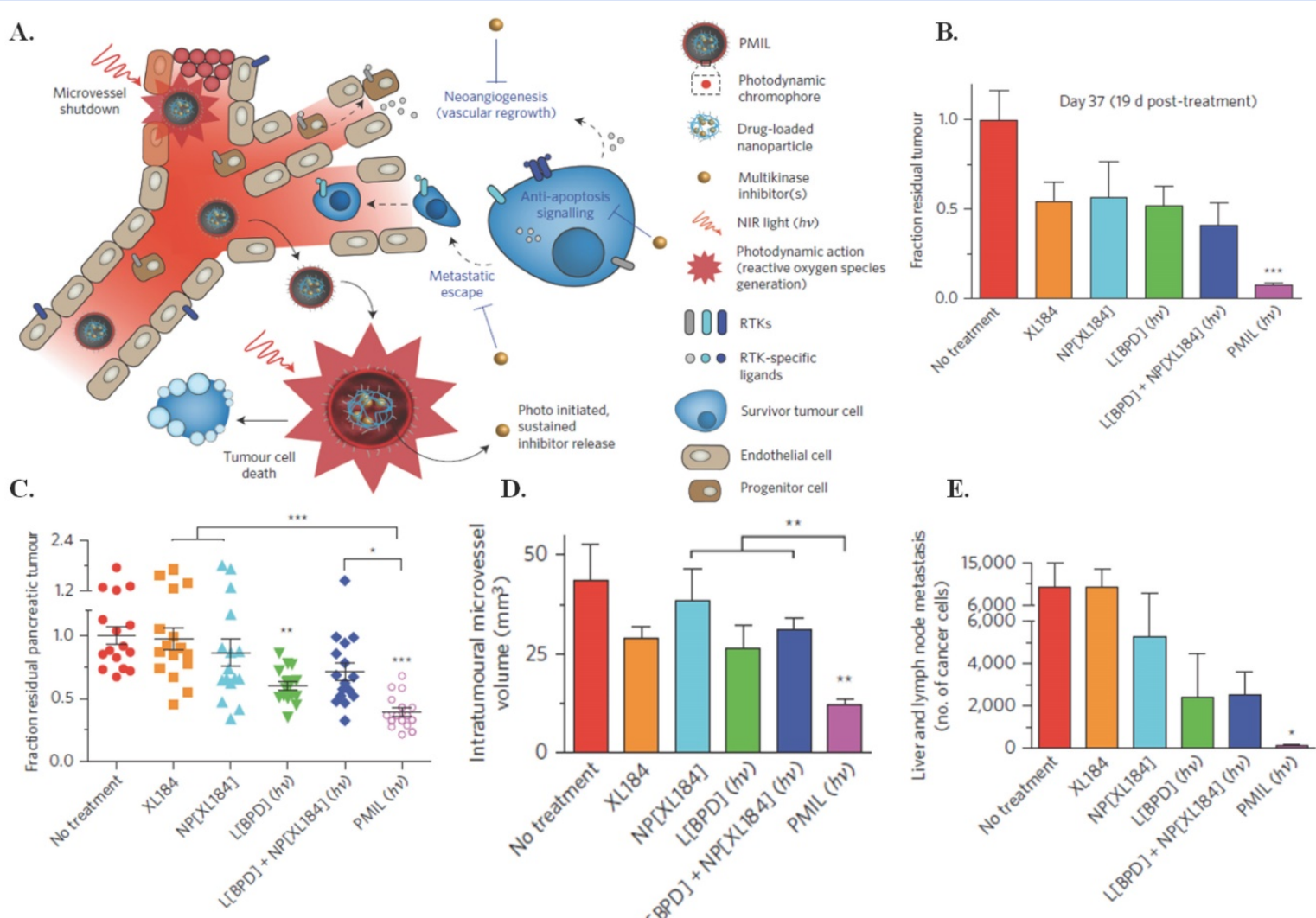

E.
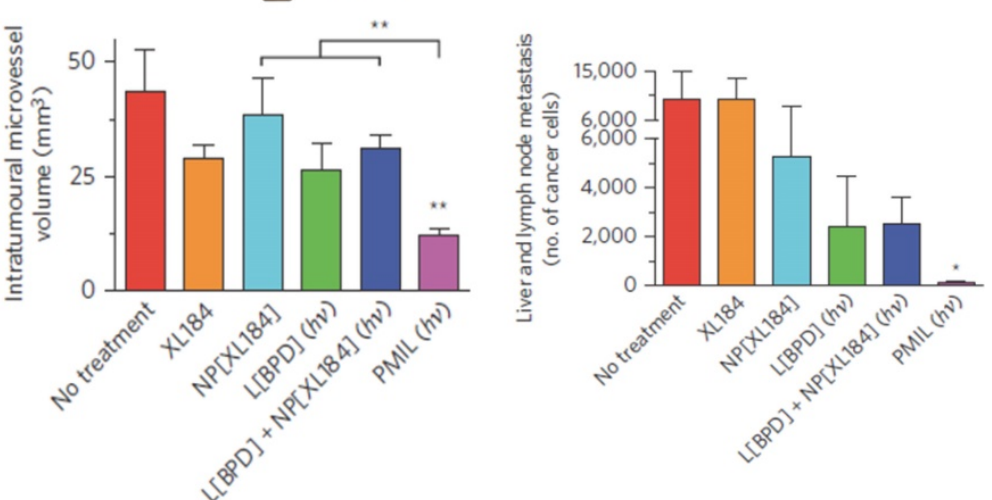

Figure 1. Photodynamic therapy using PMILs. (A) NIR light activates PMILs within the tumour microvasculature and parenchyma for simultaneous neovascular damage, tumour cell apoptosis and necrosis as well as liposome disruption with initiation of sustained multikinase inhibition. The PMIL delivery system is tunable for simultaneous delivery of photodynamic, chemotherapeutics and small-molecular inhibitors. (B) Fraction of residual tumour in a subcutaneous model $19 \mathrm{~d}$ post-treatment. Results are mean \pm s.e.m. Error is small where hidden. Asterisks in B denote significance compared with no treatment $(n=5$ mice per group; $* * * P=0.0038, \mathrm{Kruskal}-\mathrm{Wallis}$ one-way ANOVA). (C) Orthotopic model at the experiment endpoint ( $37 \mathrm{~d}$ following tumour inoculation). Results are mean \pm s.e.m. Asterisks denote significance compared with no treatment or amongst the indicated groups ( $\mathrm{n}=16$ mice per group; $* \mathrm{P}<0.05$, $* * \mathrm{P}<0.01$, $* * * \mathrm{P}<0.001$, Kruskal-Wallis one-way ANOVA). A single cycle of combination therapy using PMILs achieves enhanced reductions in orthotopic tumours $(P=0.011$, two-way ANOVA BPD-PDT $\times$ XL184 interaction term $)$ - but not using the conventional combination, $L[B P D]+$ $\mathrm{NP}[\mathrm{XL184}$ ] $(\mathrm{P}=0.23)$. (D) A single PMIL treatment induces a decrease in intratumoural microvessel volume. Results are mean \pm s.e.m. Asterisks denote significance compared with no treatment or amongst the indicated groups (no-treatment control, $\mathrm{n}=8$ entire tumour cross-section image mosaics from 8 mice; $X L 184, \mathrm{n}=7$ entire cross-sections from 3 mice; NP[XL184], $n=8$ entire cross-sections from 4 mice; L[BPD], 8 entire cross-sections from 4 mice; L[BPD] + NP[XL184], 10 entire cross-sections from 5 mice; PMIL, 10 entire cross-sections from 7 mice; **P $<0.01$, Kruskal-Wallis one-way ANOVA). (E) A single PMIL treatment reduces the total number of metastatic cancer cells in the liver and regional lymph nodes. Results are mean \pm s.e.m. Asterisks denote significance compared with no treatment (no-treatment control, NP[XL184], L[BPD] + NP[XL184], and PMIL, $\mathrm{n}=20$ mice per group; XL184, $\mathrm{n}=18$ mice; L[BPD], $\mathrm{n}=16$ mice; $* \mathrm{P}<0.05$, Kruskal-Wallis one-way ANOVA). Figure and legend reproduced from [39].

\section{Photothermal Theranostics}

Photothermal therapy utilizes continuous wave or pulsed lasers to irradiate cancer tissue with an electromagnetic radiation (e.g. visible or near infra-red light) to cause a rise in temperature that subsequently leads to cell death. Photo-absorbers convert laser energy to heat, ranging between 45-300 ${ }^{\circ} \mathrm{C}$, which can cause localized diseased tissue destruction through different mechanisms [56]. If continuous wave lasers are used for photothermal therapy, adequate energy has to be delivered in the region of interest to compensate for the heat losses that ensue in the tissue owing to blood perfusion. Pulsed lasers, on the other hand, cause intense heat to build up in the targeted tissue since the pulse width used is shorter than the typical thermal relaxation time of the tissue as per the "thermal confinement condition" [57]. This therapy, through careful selection of laser parameters and illumination, allows for specific targeting through localized light penetration. This focused targeting can significantly limit systemic effects when using photosensitive agents that are non-cytotoxic unless irradiated by a specific wavelength of light, and can ensure that the diseased tissue will respond differently from the surrounding normal tissue.

One particular application of photothermal therapy was developed to target microscopic residual disease (MRD) [16]. MRD leads to tumor resurgence and metastases even after oncosurgery. Use of plasmonic nanobubbles (PNBs), a photomechanical product of photothermal triggering, has recently proved promising in providing intraoperative detection of residual disease in vivo in real time. PNBs are generated when clusters of gold nanoparticles (conjugated to panitumumab $(60 \mathrm{~nm})$ ), are taken up via receptor mediated endocytosis and subjected to a short laser pulse that induces the formation of transient, photomechanical vapor nanobubbles. In this case, the photothermal application is for forming 
the photomechanical PNBs that can then be used for MRD detection, rather than using the photothermal effects of the gold nanoclusters for cell destruction. The synthesis mechanism can be observed in Figure 2A. These PNBs can then be used to release an acoustically detected pressure pulse within cancer cells that is different from that in normal tissues, which allows for specific targeting of cancerous tissue. Post primary tumor resection, generated PNBs can reveal in real time where MRD exists allowing for removal. In vivo application of the PNBs to gold pretreated head and neck squamous cell carcinoma (HNSCC) yielded no false-positive or false-negative signals. In unresectable MRD cases, the PNBs mechanical impact can destroy cancer cells. Unless PNBs reach a lethal size, which is determined by the

A.
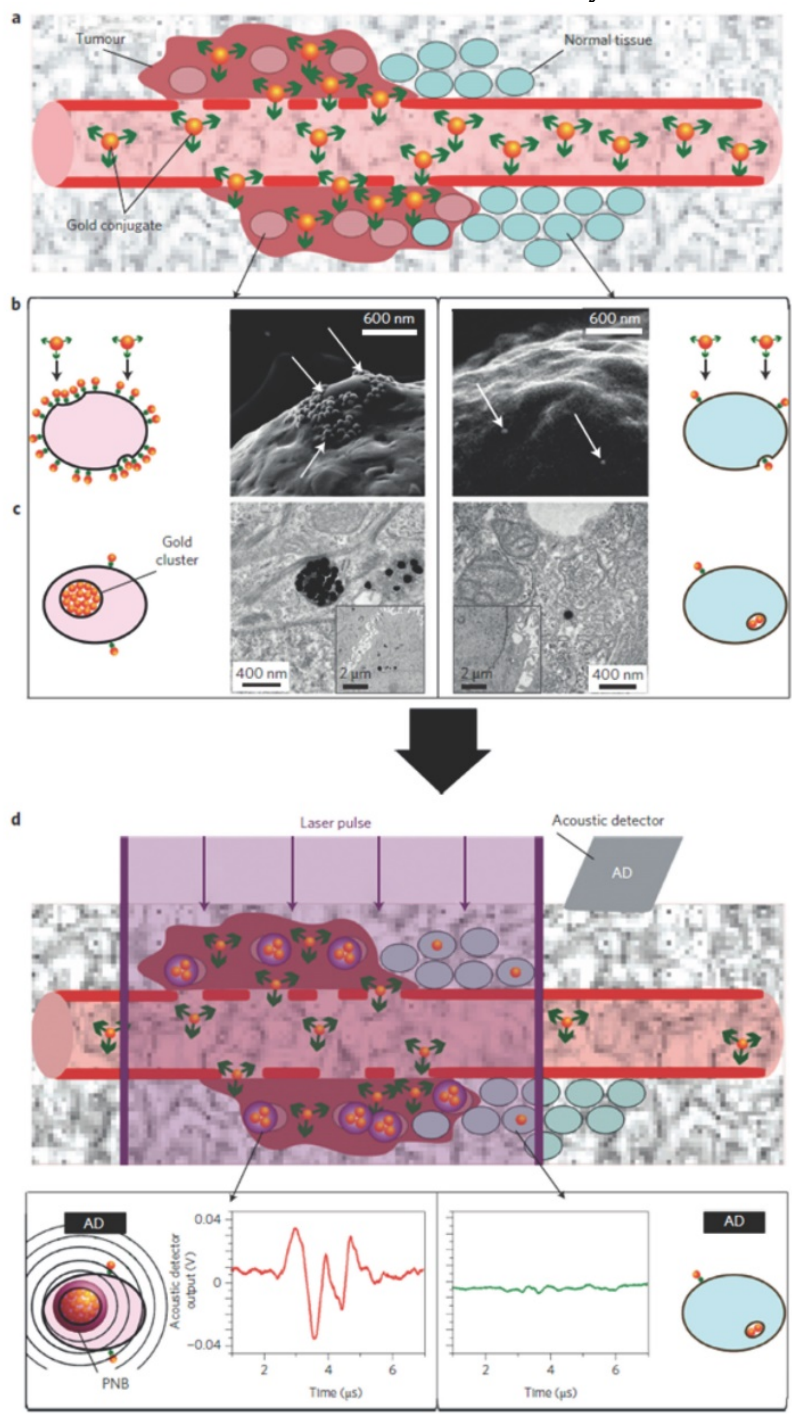

administered laser fluence [17], the method is just a diagnostic. If resection is possible, the resection continues until there is no PNB signal; $100 \%$ of the animals in this category did not experience tumor recurrence. There were no burns or damage to the surgical bed using PNBs. The PNBs have diagnostic sensitivity up to $4 \mathrm{~mm}$, and could be used clinically with a probe, on a robotic arm or surgical endoscope [16]. The acoustic output, reduction in tumor volume, and survival rates in vivo as a result of PNBS are shown in Figure 2(B-D). Earlier in vitro tests showed that PNBs may more effectively treat drug-resistant cancer cells than traditional methods by inducing a thirty times more deadly response with only a tenth of the clinical dose [18].
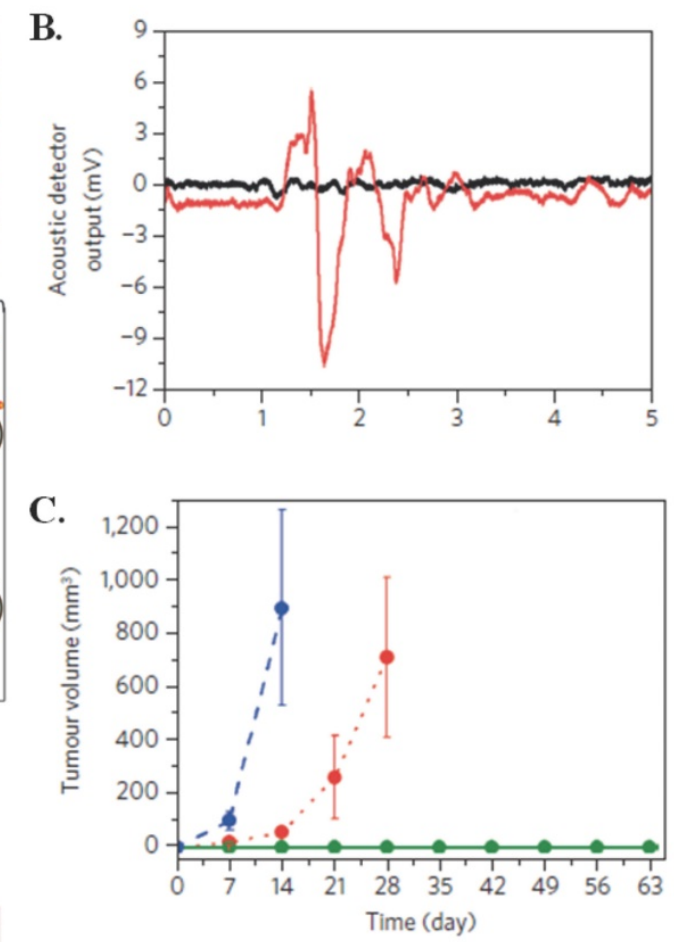

D.

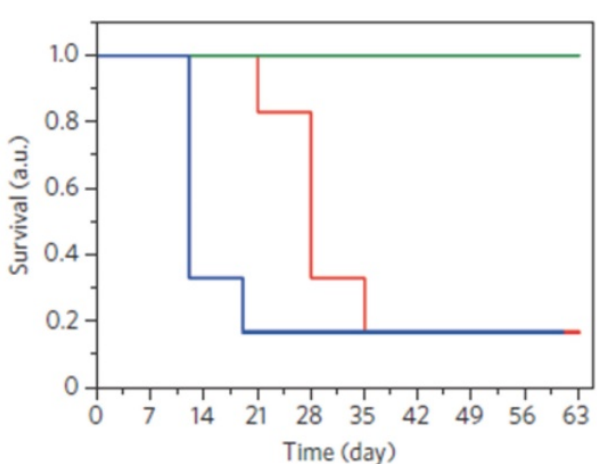

Figure 2. Photothermal effects used for forming PNBs. (A) (a) Systemic delivery of gold conjugates to the tumour via their leaky vasculature (b) Accumulation of gold conjugates by receptors of cancer cells (gold shown with white arrows in illustrative scanning electron microscopy images) (c) Intracellular clustering of gold conjugates via receptor-mediated endocytosis (illustrative transmission electron microscopy images). A gold cluster, on exposure to a single laser pulse of low fluence, selectively generates a PNB only in cancer cells; normal cells with non-specifically internalized single gold nanoparticles do not generate PNBs because of the higher threshold of PNB generation (d) The acoustic signal of a PNB (illustrative red time response) reports even a single cancer cell in solid tissue, but not normal cells (illustrative green time response). (B) Acoustic time responses before (black) and after (red) injection of ten gold conjugate-pretreated cancer cells at a tissue depth of $1 \mathrm{~mm}$. (C-D) The animal group-averaged metrics of local recurrent tumours after standard surgery with resectable MRD (blue, $n=6$ ), PNB-guided surgery of resectable MRD (green, $n=5$ ) and PNB nanosurgery of unresectable MRD (red, $n=6$ ) show a significant improvement in the outcome in both resectable and unresectable cases when the surgery is enhanced with PNBs. (C) Tumour volume (D) Animal survival. Figure and legend reproduced from [16] 
Another study using PNBs for photothermal applications focused on the dual loading of liposomes with doxorubicin (DOX) and indocyanine green (ICG). When externally stimulated by NIR (808 nm, 1 $\left.\mathrm{W} / \mathrm{cm}^{2}\right)$, DOX and ICG loaded, temperature sensitive liposomes (DI-TSL) (43.8 nm, -23.5 mV) caused hyperthermia and released DOX into mice bearing MCF-7 breast adenocarcinoma cellular endosomes, leading to cell apoptosis and tumor eradication without side effects. Nude mice treated with a single $0.5 \mathrm{mg} / \mathrm{kg}$ DOX encapsulating DI-TSL injection $(808$ $\mathrm{nm}, 0.5 \mathrm{~W} / \mathrm{cm}^{2}, 5 \mathrm{~min}$ ) showed complete tumor inhibition with no recurrence after 15 days [58].

Tumor necrosis factor-a (TNF) has also proven to be a desirable targeting agent for photothermal therapies [17, 59-62]. Polyethylene glycol-tumor necrosis factor-a (TNF)-coated gold nanoparticles (CYT-6091), a nanoparticle that has passed phase 1 clinical trials, were shown to decrease interstitial tumor fluid pressure in a 4T1 murine breast tumor model and reduce tumor growth in SCCVII head and neck tumor model [59]. Engineers have also used TNF-gold nanoparticles in conjunction with gadolinium-DTPA (Gd) to produce dynamic contrast enhanced magnetic resonance imaging (DCE-MRI) of mice bearing $\mathrm{LNCaP}$ prostate cancer [60]. These images successfully showed a non-invasive method for tracking nanoparticle delivery, retention, and vascular disruption. TNF was also targeted by another team utilizing deoxyribozyme (DNAzyme) functionalized gold nanoparticles (AuNPs) for the treatment of myocardial infarction (MI) [61]. In vivo experiments showed that Dz-AuNPs significantly reduce inflammation in MI.

Nanographene oxide particles have also been used to treat a variety of cancers. A nanographene oxide-hyaluronic acid conjugate was used to induce photothermal ablation in melanoma skin cancer using NIR light [63]. In another study, nano-sized, reduced graphene oxide-iron oxide NP (RGO-IONP) complexes that are non-covalently functionalized with polyethylene glycol (PEG) were used to image and treat 4T1 tumor bearing mice. Multimodal imaging was used to guide photothermal therapy in these animals. These nanoparticle complexes, whose preparation is illustrated in Figure 3A, exhibit strong physiological stability, NIR absorbance, and superparamagnetic properties. The structure of these nanoparticle complexes can be seen by the TEM images in Figure $3 \mathrm{~B}(\mathrm{a}-\mathrm{b})$ and the AFM images in Figure $3 \mathrm{~B}(\mathrm{c}-\mathrm{d})$. It was determined by TEM that the iron oxide nanoparticles had diameters around 8-10 $\mathrm{nm}$ and showed an even distribution on the surface of RGO. It was also determined by TEM that the sheet dimensions of RGO-IONP drastically decreased following PEGylation, with mean hydrodynamic diameters decreasing from roughly $200 \mathrm{~nm}$ before PEGylation to roughly $50 \mathrm{~nm}$ following PEGylation. The cause of this was determined to be the ultrasonication process of the PEG functionalization that broke the RGO-IONP sheets into smaller pieces. These complexes also have optimal properties for imaging, as seen in Figure 3C. The RGO-IONP complexes can be used for three different types of imaging namely magnetic resonance imaging, optical fluorescence-based imaging, and photoacoustic imaging. Additionally, these imaging methods can be combined with photothermal therapy for imaging guided photothermal tumor ablation. The effects of this irradiation are illustrated in Figure 3(D-E). It is clear that upon irradiation there was significant tumor destruction, illustrating the effects of multimodal imaging-guided photothermal therapy. These graphene nanoparticle complexes thus illustrate a novel theranostic treatment method that allows for MRI-guided photothermal therapy for tumor ablation and treatment monitoring [64].

Some additional nanomaterials used in conjunction with photothermal therapy include silicon nanowires with gold nanoparticles [65]; CuS nanoparticles [66-69]; multidye mesoporous silica with silane-conjugated heptamethine cyanine dye loaded with silicon 2,3-napthalocyanine dihydroxide dye [70]; single walled carbon nanotubes [71]; porous silicon nanoparticles [72]; NIR resonant silica core, gold shell, PEG nanoparticles [73]; chitosan-coated triangular silver nanoparticles [74]; and palladium nanosheets $[75,76]$.

\section{Phototriggered Chemotherapeutic Release}

Major disadvantages of conventional administration of drugs include an unbalanced drug release profile due to sudden changes in the biological conditions and the irregular dissemination of drugs in the human body leading to severe toxic side effects. Remotely activated delivery methods that can activate release of a drug at the appropriate location (e.g. tumor site) and at a frequency that regulates itself in response to the stage of the cancer are very appealing in oncology. Some light-responsive delivery methods are only good for one-time use. For example, the laser causes an irrevocable physical change in the system that incites a "burst" release of the payload, while others experience reversible structural changes when the laser is turned off/on and act as multi-controllable systems that release the payload in a pulsatile, on-demand routine [77]. 
A.

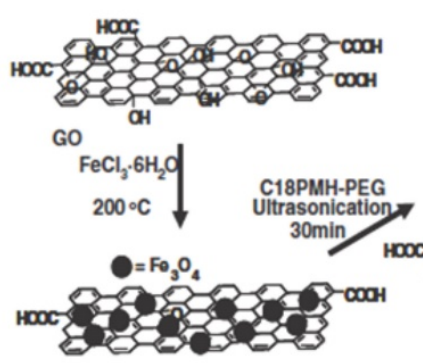

C.

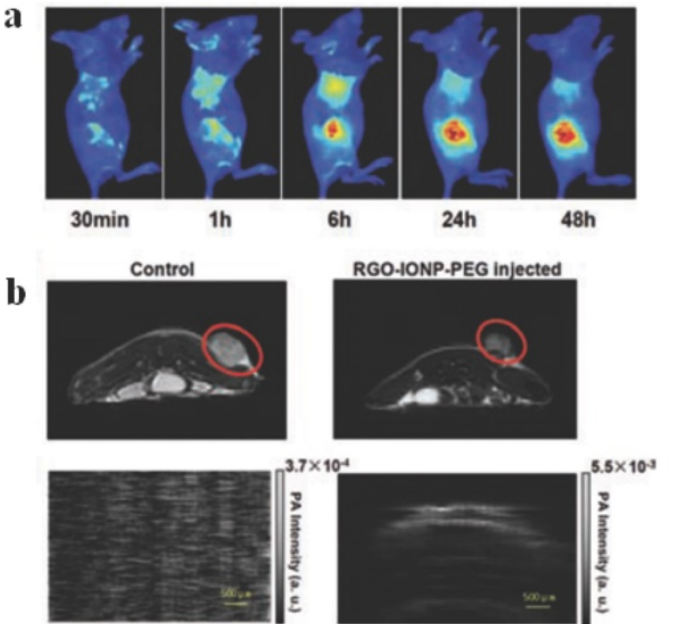

B.

a

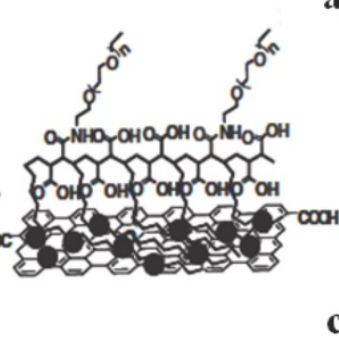

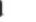

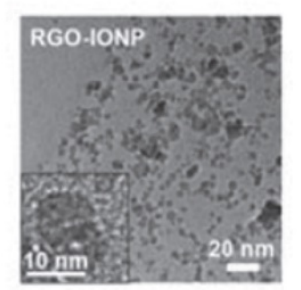

c

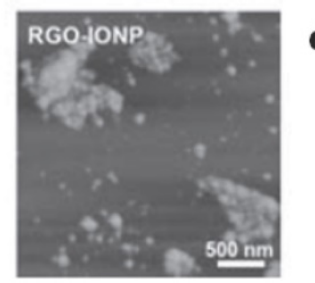

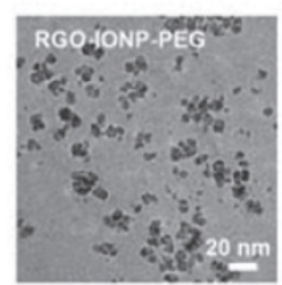

d

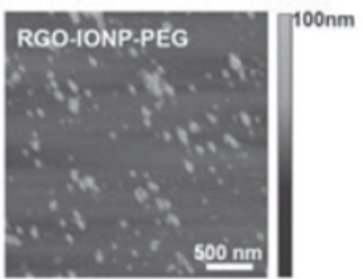

D.

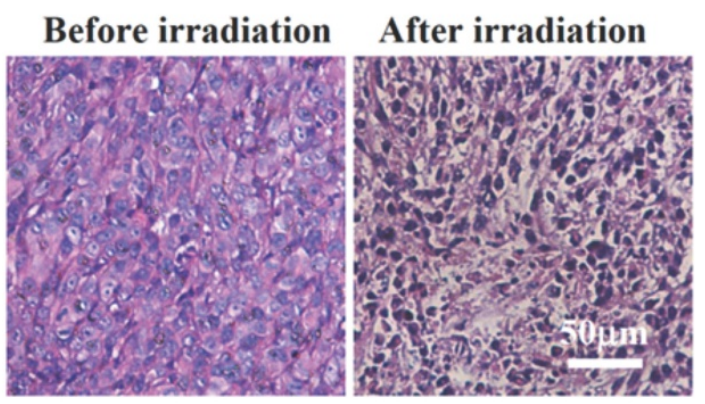

E.
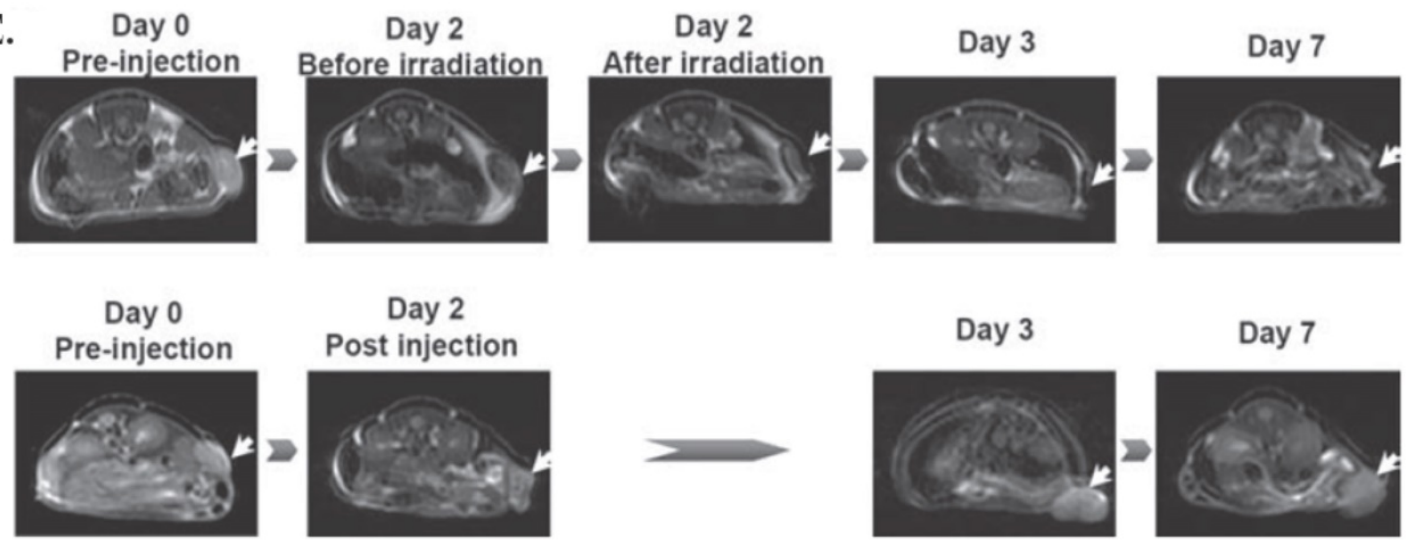

Figure 3. Imaging Guided Photothermal Therapy. (A) A scheme showing the preparation of RGO-IONP-PEG from GO. (B) (a,b) TEM images of as-made RGO-IONP (a) and RGO-IONP-PEG (b) Inset is a high-resolution TEM image of an IONP grown on the RGO sheet. (c,d) AFM images of (c) RGO-IONP and (d) RGO-IONP-PEG. The sheet size decreased significantly after PEGylation. (C) Multimodal imaging of 4T1 tumor-bearing mice after intravenous injection of RGO-IONP-PEG: (a) Fluorescence imaging using Cy5 labeled RGO-IONP-PEG; (b) T2-weigted MR imaging; and (c) Photoacoustic imaging. All the images showed that RGO-IONP-PEG could passively accumulate in the tumor after intravenous injection. (D) H\&E stained tumor slices collected from RGO-IONP-PEG injected mice before and right after laser irradiation. (E) MR images of RGO-IONP-PEG injected mice with (the upper low) and without (the lower row) laser irradiation. Arrows point the tumor sites. Images were collected at day 0 (pre-injection), day 2, day 3 and day 7 p.i. Both tumor growth curves and MR imaging data showed that tumors on mice injected with RGO-IONP-PEG and irradiated with the NIR laser were completely ablated, while the tumor growth of control mice was not affected. Figure and legend reproduced from [64].

Engineering a non-invasive drug delivery method with increased tumor penetration and limited toxicity is essential for the improved chemotherapeutic treatment of cancer. Tong et al. constructed a docetaxel encapsulating nanoparticle comprised of spiropyran, polyethylene glycol, and cholesterol (Dtxl/SP $\mathrm{NP}_{\mathrm{HC}} \mathrm{S}$ ) that, when triggered by $365 \mathrm{~nm}$ light, undergoes a size change from 103 to 49 $\mathrm{nm}$ that allows for increased tumor penetration and simultaneous drug release intratumorally via intravenous administration in a subcutaneous implanted fibrosarcoma model [82]. This mechanism and size change, as a result of irradiation by $365 \mathrm{~nm}$ light, can be seen in Figure 4(A-B). Ex vivo application of the photoswitching nanoparticle through skin demonstrated a half time of 25.9 seconds at $1 \mathrm{~W} / \mathrm{cm}^{2}$. 
Intratumoral injection of Dtxl/SP $\mathrm{NP}_{\mathrm{HC}} \mathrm{S}$ in mice demonstrated notable tumor reduction, as seen in Figure $4 \mathrm{C}$, and insignificant changes in body weight for 15 days; three of five mice treated survived more than 100 days, two of which had complete tumor resolution. There was also observed tumor reduction and longer survival times when Dtxl/SP $\mathrm{NP}_{\mathrm{HC}}{ }^{\mathrm{S}}$ were injected intravenously (Figure 4(D-E)). As seen by the histological analysis in Figure $4 \mathrm{~F}$, the greatest antitumor effect occurs when Dtxl/SP $\mathrm{NP}_{\mathrm{HC}} \mathrm{S}$ are combined with light, causing a size change. Additionally, the nanoparticles did not affect blood tests, and proved to have limited toxicity. The treatment mechanism causes increased apoptosis, tumor vessel decompression, and decreased cell proliferation as a result of light-triggered penetration and drug release. [78]

A.

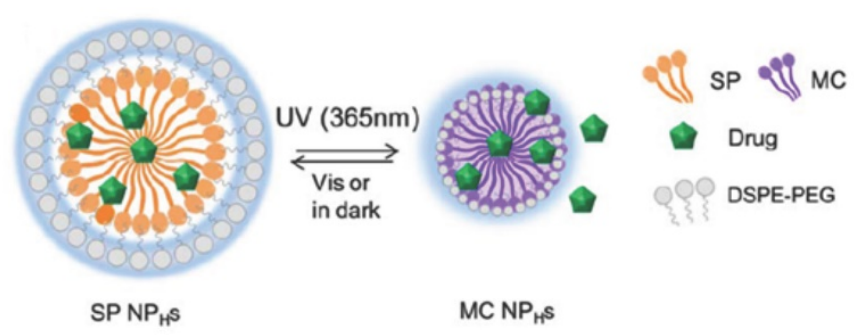

B.

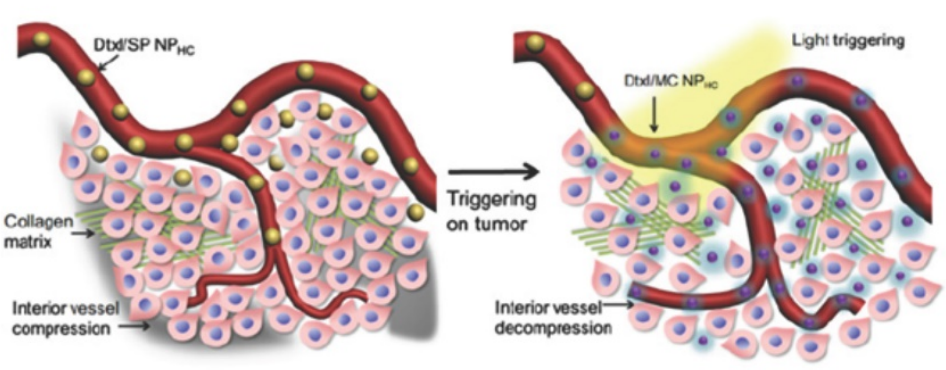

C.

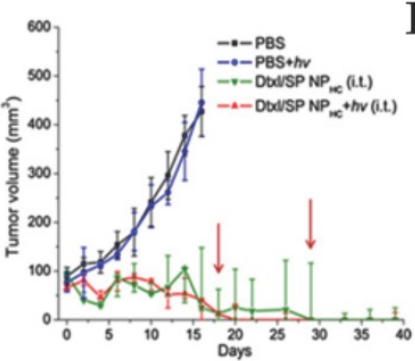

E.

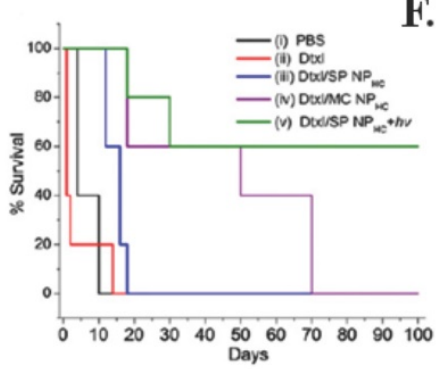

D.

F.
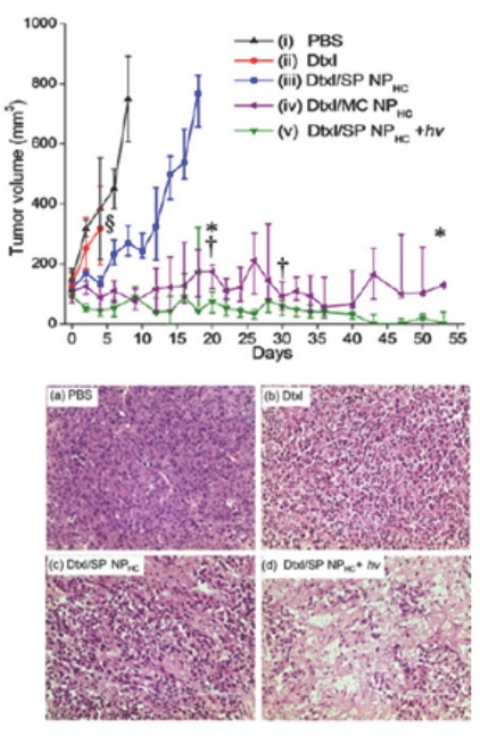

Figure 4. Phototriggered chemotherapeutic release. (A) Scheme of photoswitchable SP / DSPE-PEG lipid hybrid nanoparticles (NPHCS). (B) Effects of light triggering of DtxI/SP NPHCS (yellow spheres) in the tumor vasculature. The dense collagen matrix (green lines) and compressed vessels prevent unshrunken NPHCS from delivering drugs within the tumor (gray region). Tumor irradiation shrinks NPHCS (purple spheres), which enhances their penetration through the collagen matrix throughout the tumor. The triggered released of Dtxl (the cyan glow surrounding $\mathrm{NP}_{\mathrm{HCS}}$ ) kills tumor cells, which leads to dilation of compressed intratumoral blood vessels. The decompression of vessels facilitates the transport of NPHCS into the tumor interior. (C) In vivo efficacy of Dtxl/SP NPHC (Dtxl dose: $10 \mathrm{mg} / \mathrm{kg}, \mathrm{n}=5$ ) given i.t. to s.c. HT-1080 tumors, without or with light triggering $\left(15 \mathrm{~s}, 1 \mathrm{~W} / \mathrm{cm}^{2}\right)$. Two of five mice in the Dtxl/SP NPHC group (green line) were euthanized as their tumor size exceeded $500 \mathrm{~mm}{ }^{3}$ (tumor diameter over $1 \mathrm{~cm}$ ) on day 18 and 29 (red arrows). Data are medians \pm quartiles. (D) Effect on tumor growth inhibition (Dtxl dose (intravenously): $40 \mathrm{mg} / \mathrm{kg}$, $\mathrm{n}=5$ ). $\S$ indicates study termination for the group treated with Dtxl due to significant body weight loss ( $>20 \%)$. Mice with tumor volumes over $500 \mathrm{~mm}^{3}$ were removed from the study (*, from the Dtxl/MC NPHC group; $\dagger$, from the Dtxl/SP NPHC group with irradiation). (E) Kaplan-Meier plot for the same groups as in C. (F) Histological analysis of HT-1080 tumor tissues following different treatments (a-d) Representative hematoxylin and eosin stained sections of tumor tissues collected from animals $96 \mathrm{~h}$ after intravenous injection of (a) PBS; (b) free Dtxl (10 mg/kg); (c) Dtxl/SP NPHC (40 mg/kg) and (d) Dtxl/SP NPHC + hv (40 mg/kg). Scale bar: $100 \mu \mathrm{m}$. Figure and legend reproduced from [78]. 
Another promising nanoparticle, this time for the treatment of cervical cancer, is a silica core graphene shell structure with a conjugated serum protein and folic acid on the graphene shell, and loaded doxorubicin $\left(\mathrm{SiO}_{2} @\right.$ GN-Serum-FA-Dox) [83]. The combined effect of the photothermal ablation with the release of doxorubicin in vivo showed no tumor regrowth over the 19-day study and significantly decreased tumor activity $(0.28 \pm 0.13 \mathrm{~g})$ compared to the control $(2.0 \pm 0.20 \mathrm{~g})$ and $\mathrm{SiO}_{2} @ G N-S e r u m-F A$ nanoparticles $(0.74 \pm 0.22 \mathrm{~g})$. Given that this nanoconstruct can specifically target cancerous overexpression of folate receptors, the particle may be applicable to other cancer types, i.e. breast or pancreatic cancer. Additionally, the photothermal effect of the base nanoconstruct shows promise for other biomedical applications [79].

Chemophotoherapy, the combination of a phototherapy (i.e. photodynamic or photothermal therapy) with chemotherapeutic release, has also been beneficial specifically in the treatment of solid tumors. By combining a phototherapy with a chemotherapeutic agent, the chemotherapeutic can increase the efficacy of the phototherapy by inhibiting tumor growth and killing off any surviving cancer cells left after photo-treatment [80]. One example includes porphyrin-phospholipid liposomes loaded with doxorubicin. These liposomes were ineffective means of treatment when only phototriggered chemotherapeutic release or only phototherapy was used, but when combined in a single chemophototherapy there was effective tumor eradication [81]. This is just one additional example of the possible uses of phototriggered chemotherapeutic release for the treatment of cancerous tumors.

Additional chemotherapy encapsulating nanoconstructs that show initial promise for the treatment of cancer include gold nanorod-cored biodegradable micelles [82], coumarin-functionalized block copolymers [83], photodegradable polyurethane self-assembled nanoparticles [84], bismuth selenide nanocomposites [85], gold nanospheres [86], PLGA coated with pH-responsive NAcHis-TPGS [87], $\mathrm{Cu}_{1.75} \mathrm{~S}$ nanocapsules [88], aptamer/hairpin DNA gold nanoparticle [89], PEGylated liposomes [81], graphene oxide nanocarriers [90], gold nanorods [91, 92], plasmonic nanoparticles [89, 93], C225-conjugated hollow nanospheres [94], and other hybrid nanoparticles [95-102].

\section{Ultrasound-Triggered Theranostics}

Ultrasound is used considerably in medicine for a variety of diagnostic and remedial purposes, including medical imaging, physiotherapy and surgery. It was proposed as a method for triggering drug delivery by Langer and co-workers in 1989 [103]. Recently, ultrasound has been explored as a means of exerting external control in drug delivery from biomaterials for pulsatile release. The advantage of ultrasound is that it is non-invasive and may be focused at depth in soft tissue throughout the body [31]. Ultrasound is an attractive mechanism for delivery of proteins, especially insulin, as it offers a reproducible, rapid and reversible method of controlling release with no degradation of proteins. The biological effects of ultrasound can cause formation of cavitation bubbles, localized tissue heating, and radiation force, which can be used for confined drug release from nanosystems, increased extravasation of drugs and/or nanoparticles from blood vessels into tumors, and improved penetration of drugs into tumors [32]. When using ultrasound for temperature sensitive nanosystems, the encapsulated payload can be released locally through the use of these mechanical forces.

Multispectral optoacoustic tomography (MSOT) is a potential, non-invasive alternative to clinically approved sentinel lymph node (SLN) excision, which is an invasive and currently accepted method for diagnosing melanoma, the fifth most common cancer in the United States [33]. MSOT utilizes ultrasound with fluorescent dyes to provide real time images of melanin in SLN to improve detection and treatment. MSOT ex vivo studies showed $100 \%$ sensitivity and $62 \%$ selectivity. In vivo use of indocyanine green (ICG) administered peritumorally in conjunction with pulse echo ultrasound imaging, 2D MSOT detected SLN at different depths - inguinal $(\sim 5 \mathrm{~mm})$, cervical $(\sim 10$ $\mathrm{mm})$, and axillary $(\sim 25 \mathrm{~mm})$. A schematic of the injection method and the images from the 2D MSOT detector can be seen in Figure 5. MSOT with ICG proved to be effective at detecting SLN up to a $50 \mathrm{~mm}$ depth with $100 \%$ sensitivity and $48.6 \%$ specificity in vivo. This method shows great clinical promise for a non-invasive single step for determining metastatic potential and node morphology with zero false negatives. Sources contributing to false positives, where melanin signals were detected but there was no metastasis, include hemorrhaging, pigmented cells, tattoo pigment, or other spectral absorbers. The test could be improved through more accurate targeting of melanoma cells. Additionally, this unique use of ultrasound can be taken further by incorporating a chemotherapeutic agent into a nanostructure along with ICG, therefore adding a therapeutic effect to the already successful diagnostic applications of MSOT [32]. The maximum depth penetrated by optoacoustic tomography using long wavelength $(>1 \mathrm{~cm})$ contrast agents is greater than $11 \mathrm{~cm}$ in tissue and $5 \mathrm{~cm}$ in a healthy living human [104]. 


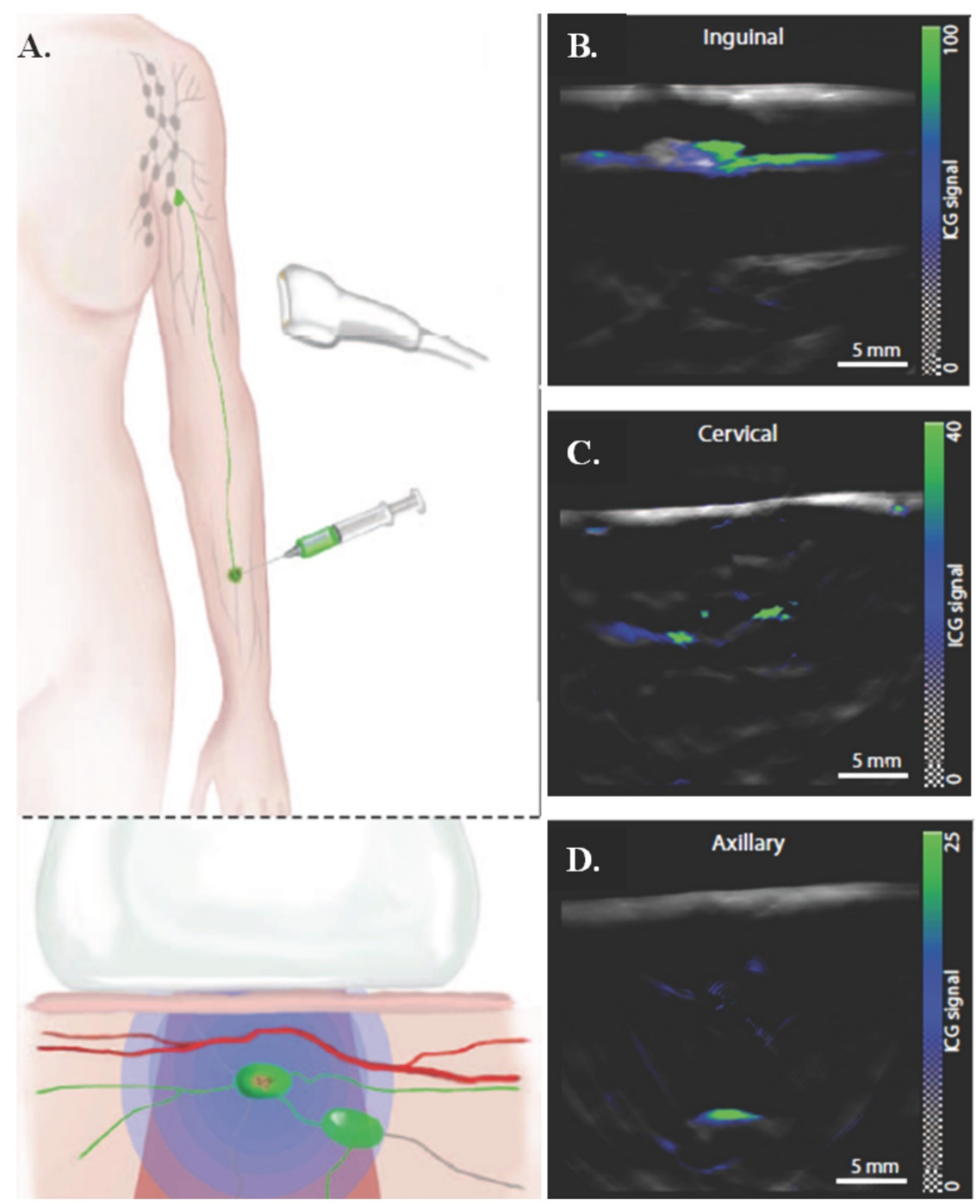

Figure 5. Multispectral optoacoustic tomography for determining metastatic status of sentinel lymph nodes in melanoma. (A) Schematic of an ICG injection for SLN detection and the generation and detection of photoacoustic signals. ICG indicates position of SLNs and in conjunction with a handheld 2D MSOT detector can indicate the location and metastatic status of the lymph node. (B-D) Representative preoperative images of inguinal, cervical, and axillary SLNs from the 2D detector, with ICG signal overlaid on a single-wavelength background image at $800 \mathrm{~nm}$. Images are from three different patients. Figure and legend reproduced from [33].

Promising cancer therapies using ultrasound include polypyrrole hollow microspheres [105], microbubbles [106-109], temperature sensitive liposomes [110-114], biodegradable poly(methacrylic acid) based nanocapsules [115], superparamagnetic iron oxide acoustic droplets [116], crown-ether-coated core/shell nanoparticles [117], polymer-grafted mesoporous silica nanoparticles [118], and echogenic glycol chitosan nanoparticles [119].

In one such study, low temperature sensitive liposomes (LTSLs) loaded with chemotherapeutic drug, doxorubicin (DOX), were investigated for image-guided treatment in rabbits [114]. These DOX loaded liposomes were used in combination with magnetic resonance-guided high intensity focused ultrasound (MR-HIFU) and were tested in vivo in rabbits with Vx2 tumors, a well-studied rabbit tumor model that is representative of a number of human cancers like those of breast, neck, lung, liver, bladder, and kidney. An experimental timeline of the image guided hyperthermia can be seen in Figure 6A and the in vivo planning and mapping for the image guided hyperthermia is seen in Figure 6B. The temperature of the image guided hyperthermia was monitored over time and over the area of treatment (Figure 6C). This study found that the combination of LTSLs with MR-HIFU resulted in up to 3.5-fold more DOX delivery to the tumor compared to drug loaded LTSLs 
and up to 7.6-fold more DOX delivery compared to free DOX. These results demonstrate the positive effects of combining drug loaded liposomes with a form of ultrasound. Additionally, it was also found that LTSLs in combination with MR-HIFU resulted in 43-fold higher DOX concentration in the tumor over adjacent muscle tissue, illustrating target-specific aspects of the treatment method. The comparative effects of free DOX, LTSLs, and LTSLs in combination with MR-HIFU can be seen in Figure 6D. Overall, this study demonstrated the benefits of an image guided treatment method by illustrating the increased bio-distribution of DOX from a liposomal construct in combination with MR-HIFU [114], which could form the basis for a clinical trial using such image-guided nanotheranostic platforms.

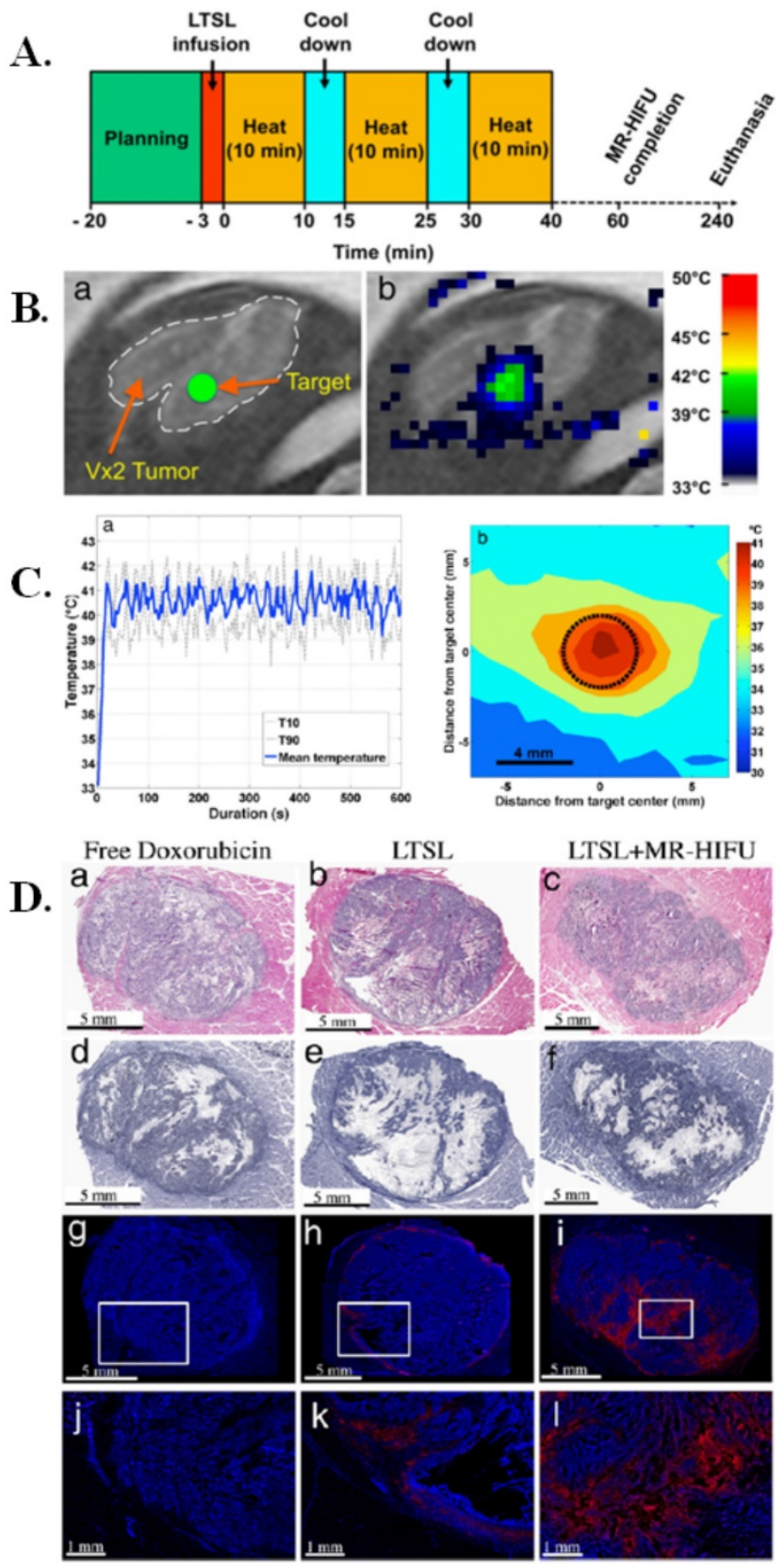

Figure 6. Image-guided drug delivery with magnetic resonance guided high intensity focused ultrasound and temperature sensitive liposomes. (A) Schematic representation of MR-HIFU experimental time line for image guided hyperthermia. Following acquisition of planning images and a slow infusion of LTSL, hyperthermia $(10 \mathrm{~min})$ was interleaved with 5 -min cooling periods. This was repeated for a total of 3 treatments or until 30 min of heating was achieved within $1 \mathrm{~h}$ after drug infusion. Rabbits were euthanized $4 \mathrm{~h}$ after LTSL infusion and tissues were harvested for HPLC or histological analysis. (B) Planning and temperature mapping for image-guided hyperthermia. (a) The $\mathrm{V} \times 2$ tumor was clearly identified on the planning images and a treatment target (diameter $=4 \mathrm{~mm}$ ) was placed in the middle of the tumor (green circle), avoiding bone, vessels and fascial planes when possible. (b) Real-time temperature monitoring using the proton resonance frequency shift method shown in color overlaid on the planning image (grayscale). (C) Image guided hyperthermia. Representative examples of temperature elevation and spatial distribution during a sonication. (a) Following a short heat-up period $(\sim 20 \mathrm{~s})$, stable mild hyperthermia was achieved in the target region through binary feedback control. (b) Time averaged spatial distribution of temperature in the target region (black circle) and the surrounding tissue, showing a uniformity of elevated temperature in the target region. (D) Histological and fluorescence analysis of $V \times 2$ hindlimb tumors following treatment. $(a-c)$ H\&E staining of tumor encased in muscle; $(d-f) \mathrm{NADH}$ viability staining of tumors (viable=blue/purple, clear/white=cellular death); (g-l) Fluorescence images of doxorubicin distribution with location of higher magnification shown by the box (nuclei=blue and doxorubicin=red). Figure and legend reproduced from [114]. 


\section{Electro-Thermally Triggered Theranostics}

Electrical signals are fairly easy to create and regulate, making them excellent remote triggers for theranostic applications. Electrical stimuli have already been effectively used to activate the release of payloads through the use of conducting polymers or implantable electrical delivery systems [34, 120, 121].

One version of an electrically stimulated drug delivery system is the use of another living organism.
"Bacteriobots," constructed with S. typhimurium and conjugated to Cy5.5- coated polystyrene microbeads using biotin and streptavidin, allow for improved targeting because of greater motility and velocity towards tumor cells as compared to "normal" cells, which can aid in fluorescent imaging [122]. The structure of these bacteriobots is illustrated in Figure 7A and a confocal image of the bacteriobots can be seen in Figure 7B.

A.

B.
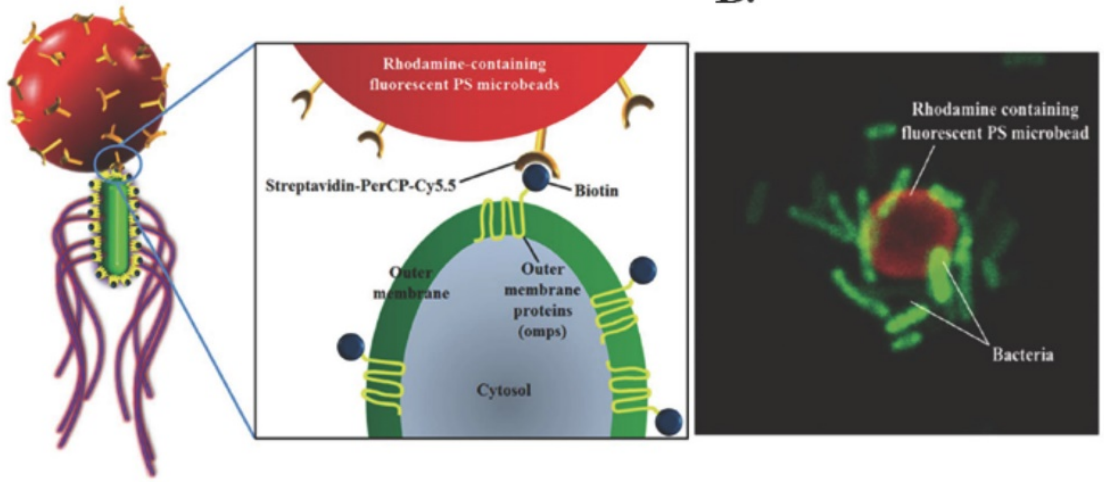

C.
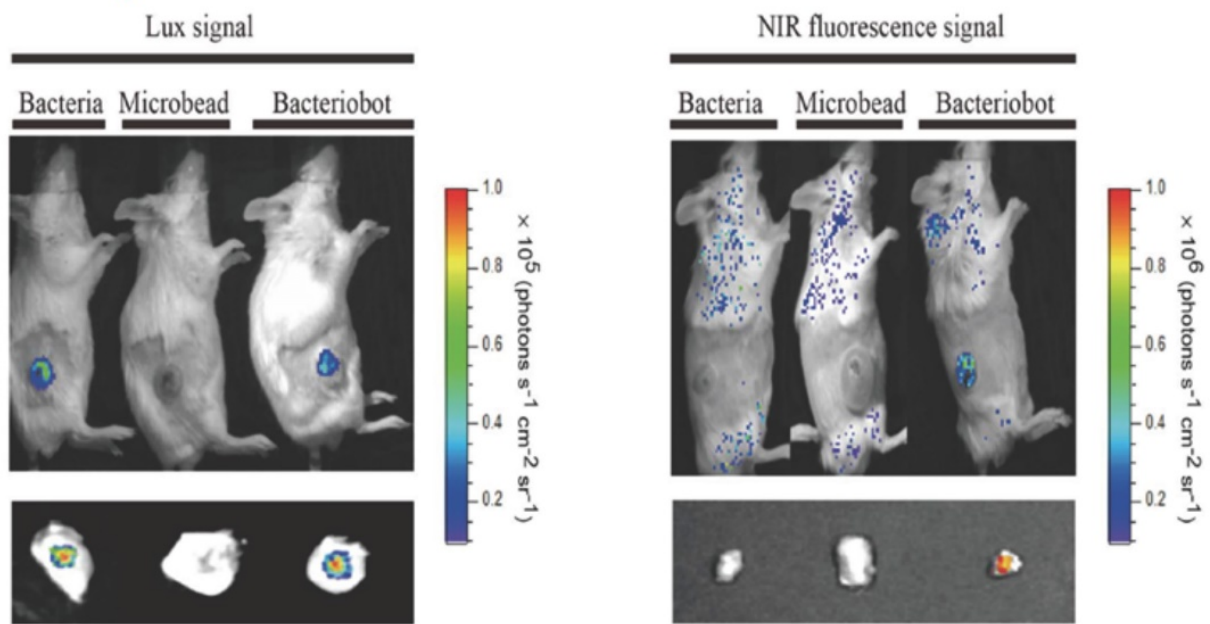

E.
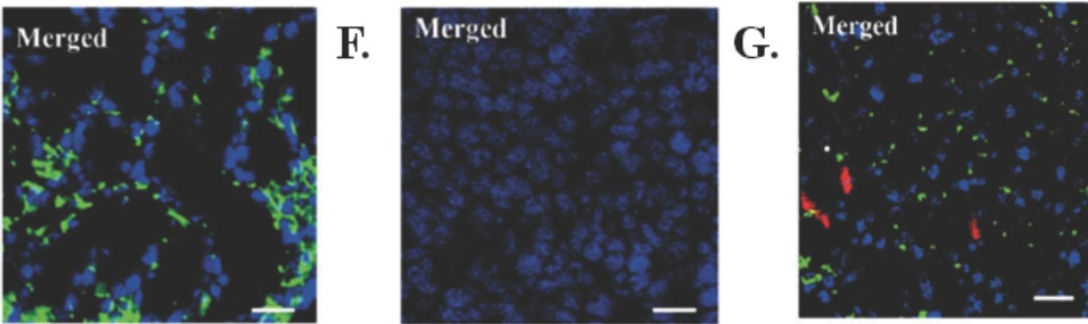

Figure 7. Bacteriobots for electro-thermally triggered theranostics. (A) Schematic representation of bacteriobots. Biotin (500 $\mu \mathrm{g})$ was incubated with omp-expressing S. typhimurium $\left(3 \times 10^{8}\right.$ cells $\left./ \mathrm{mL}\right)$ for $1 \mathrm{hr}$. Rhodamine-containing fluorescent carboxylated PS microbeads $\left(1 \times 10^{8} / \mathrm{ml}\right)$ were covalently coupled to streptavidin-PerCP-Cy5.5 (500 $\mu \mathrm{g})$. Biotin-displaying S. typhimurium and streptavidin-PerCP-Cy5.5-coated PS microbeads were co-incubated for 30 min at $37^{\circ} \mathrm{C}$. (B) S. typhimurium-attached PS microbeads were observed using a confocal laser scanning microscope. (C-D) Mice (n56) were injected subcutaneously with CT-26 cells $\left(1 \times 10^{6}\right)$. When the tumors reached a volume of approximately $130 \mathrm{~mm}^{3}$, the tumor-bearing mice were injected with bacteria $\left(3 \times 10^{7} \mathrm{CFU} / 100 \mu \mathrm{L}\right)$, microbeads $\left(1 \times 10^{7} / 100 \mu \mathrm{L}\right)$ or bacteriobots $($ bacteria:microbeads ratio $=$ 3:1 per $100 \mu \mathrm{L}$ ). Representative in-vivo and ex-vivo bioluminescence and NIR images (Cy5.5 image) were captured 3 days post-injection. (C) In-vivo bioluminescence and NIR imaging of mouse tumor models. (D) Ex-vivo bioluminescence and NIR imaging of the dissected tumors. (E-G) Tumor-bearing mice were injected with (E) bacteria, (F) microbeads, and $(\mathbf{G})$ bacteriobots, the tumor masses were fixed and investigated histologically, and bacteria, microbeads, and bacteriobots were localized by indirect fluorescence. DAPI staining of the same tissue sections and mergence of the DAPI-stained slides (blue); bacteria were detected by indirect immunofluorescence (green); microbeads and bacteriobot were detected by indirect fluorescence (red) in the dissected tumor masses. Scale bars, $10 \mathrm{~mm}$. Figure and legend reproduced from [122]. 
The chosen bacteria strain is easily genetically manipulated, can direct chemotaxis to certain molecular signals through chemotactic receptors, has high-motility flagella that respond to external chemical stimuli, and has been shown to target and proliferate in solid tumors through self-propulsion that aids in penetration. Subcutaneous injection of the bacteriobots in CT-26 tumor-bearing mice demonstrated successful targeting via bioluminescence, as seen in Figure 7(C-D). In addition, as seen in Figure 7(E-G), the bacteriobots showed fluorescence in Cy5.5 (red) and there was promising accumulation of bacteriobots in tumors 3 days after infection. Next steps for this research include using the bacteriobots for payload delivery using appropriate cancer models [122].

To circumvent the limitations of conventional electric stimuli-responsive drug delivery devices, Zare and co-workers recently described a novel dual-stimulus responsive nanosystem for externally triggered payload delivery [123]. This nanosystem was capable of being triggered by temperature changes and use of an electric field. Polypyrrole nanoparticles were loaded with therapeutics and then subcutaneously localized in vivo with the help of a temperature-sensitive hydrogel using a triblock polymer, PLGA-PEG- PLGA. Drug delivery from the conductive nanoparticles could be controlled by use of a weak, external electrical trigger. This approach exemplifies an innovative and interactive nanosystem that can be externally activated with decent/satisfactory/acceptable control on the time, location, and amount of drug released [123].

Other electro-stimulated systems that could prove promising for cancer applications include sensing electrodes in an alginate matrix [124], electrical impedance sensing system [125], iron-alginate thin-films [126], electro-active hydrogel based polymer matrix [127, 128], gold nanoparticles activated by light and an electric field [129], nanowire substrates [130], and platinum microelectrodes [131].

\section{Magneto-Thermally Triggered Theranostics}

Initial experiments with magnetically regulated drug delivery systems used fairly large magnetic beads in the millimeter size range that had to be implanted in an ethylene vinyl acetate milieu. These large beads could be activated through the use of an oscillating magnetic field to open pores for drug release. This approach, however, led to very few real applications in drug delivery due to very slight differences between the "on" and the "off" states [132, 133].
Recent progress in nanomedicine has led to experimenting with magnetic nanoparticles, which can be heated by using an alternating current (AC) magnetic field. Use of such magnetic nanoparticles caused a renewed interest in this field of triggered drug delivery. Most of the current work focuses on magneto-thermally-responsive or magneticallytriggered, thermally-sensitive nanomaterials for drug delivery applications. Two fundamental measures are required for magneto-thermal delivery: magnetic heating or magnetic hyperthermia, and a temperature-responsive or thermally-rupturable layer. Once the activation is complete, the advantage of regulating drug release helps the patient by decreasing the net quantity of drug needed to achieve efficacy and by reducing the number of administrations required. Such magnetically triggered systems could be incorporated into more refined devices that comprise targeting moieties, imaging agents and multi-modal therapy. [134]

To overcome delivery and toxicity issues associated with pancreatic cancer, Lee et al. developed urokinase plasminogen activator receptor (uPAR)-targeting iron oxide nanoparticles (IONPs) loaded with gemcitabine (Gem) (ATF-IONP-Gem) to image and treat uPAR-expressing tumor and stromal cells [6]. As seen in Figure 8A, the Gem is conjugated to the IONP using an amino terminal fragment (ATF) so that it is only released after lysosomal or endosomal enzymatic cleavage via receptor-mediated internalization. In addition to delivery of Gem, the IONPs have proven to be an acceptable method for MRI imaging (Figure 8B). The ATF-IONP-Gem nanoparticles (hydrodynamic size $66 \mathrm{~nm}$ ) yielded 50\% tumor growth inhibition in an orthotopic human pancreatic cancer xenograft model as observed in Figure 8C. Post ATF-IOP-Gem treatment (Figure 8D) there was no evident tumor growth or Ki-67 expression, a marker of cell proliferation. The nanoparticle did not damage the liver, spleen, or other organs. These results are promising for in vivo applications given the effective targeting, and drug conjugation stability. [6]

Drug release strategies using nano-sized hybrid membranes that contain temperature-responsive nanogels and superparamagnetic nanostructures have been shown to afford reversible "on/off" payload release upon the application and elimination of an alternating magnetic field [135]. Kohane and co-workers show that altering the phase change temperature of the nanogel could regulate the dose of drug delivered across the membrane. Other factors that could influence the dose delivered include the number of nanogels loaded in the membrane as well as the thickness of the membrane. Such membranes 
allow for triggered and regulated delivery of model drug mimetics over about two orders of magnitude ranging from 0.1 to $10 \mu \mathrm{g}$ drugs/h. The drug release kinetics across the membranes permit drug doses to be tuned according to the time period of the magnetic field. Therapeutics over a wide range of sizes (0.5-40 $\mathrm{kDa}$ ) could be delivered by using this device [141]. The authors clearly demonstrate reproducibility with their devices by changing membranes and altering cycles, thereby signifying the universal value of these membranes for anti-cancer treatments. Rigorous testing and characterization of these constructs still has to be carried out before they can be used for clinical applications [136].

Iron oxide nanoparticles (IONPs) are a commonly chosen delivery mechanism for magnetically triggered therapies; integration of IONPs on a M13 filamentous bacteriophage scaffold were used treat prostate cancer [137], IONPs aided in wireless deep brain stimulation [138], IONPs integrated with polymerosomes were used to treat HeLa cells [139], IONPs were combined with lipid membrane vesicles to form a drug delivery system

A.

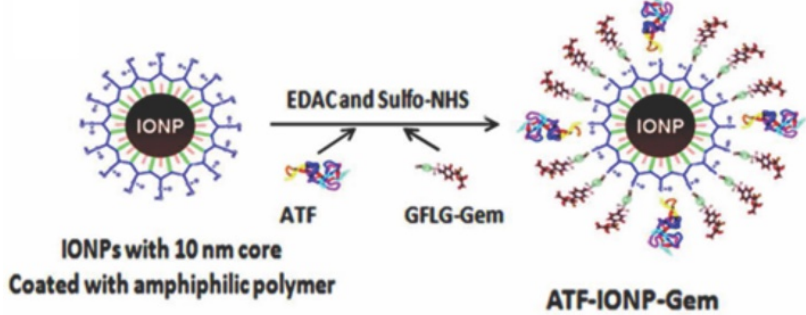

C.

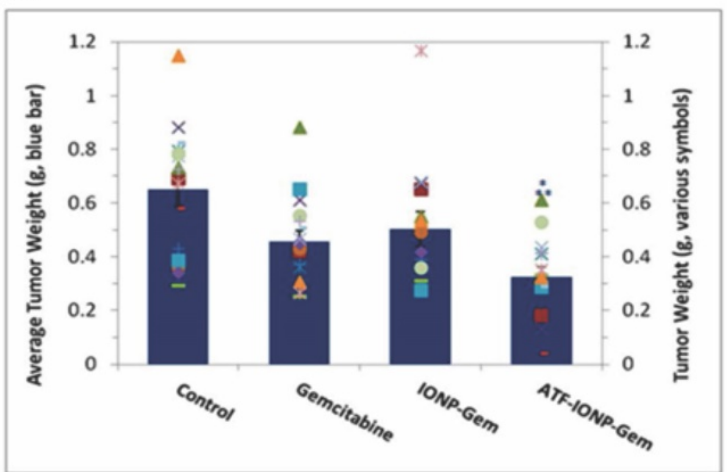

[140], and clusters of superparamagnetic IONPs with folic acid and PEG were shown to have specific accumulation in tumors and enhanced MRI contrast [141]. Another material that has been used with magneto-thermal triggering is zinc ferrite spinel-graphene nanostructures [142].

\section{Additional Remotely Triggered Treatments}

\section{X-Ray Irradiation}

Another possible remotely triggered treatment includes X-ray induced ionizing radiation. A use of $\mathrm{X}$-rays is to induce the generation of a reactive oxygen species like in photodynamic therapy. One study focused on $\mathrm{CeF}_{3}$ nanoparticles conjugated with the photosensitizer verteporfin for the generation of a reactive oxygen species upon $8 \mathrm{keV} \mathrm{X}$-ray irradiation. The results produced similar singlet oxygen molecules per cell as compared to photodynamic therapy. This method may be used either as an independent treatment method or as a supplement to radiation treatment [143].

B.

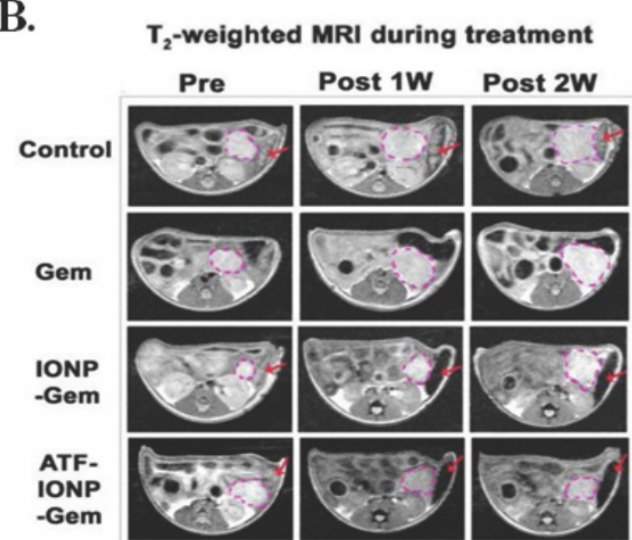

D.

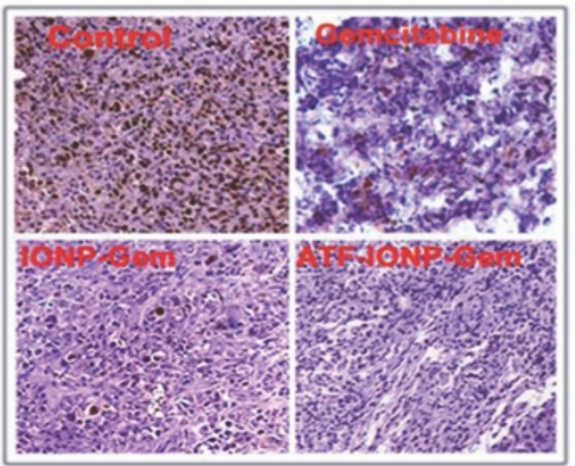

Figure 8. IONPs for magneto-thermally triggered theranostics. (A) Diagram of the conjugation of ATF peptides and GFLG-Gem conjugates to IONPs. (B) Coronal T2-weighted MR images and corresponding bright field (BF) images of the tumor-bearing mice after systemic delivery of non-targeted IONP-Gem or ATF-IONP-Gem. Tumor bearing mice without nanoparticle treatment were used as controls. Yellow dotted circles and arrows indicate the location of primary tumor lesions in the MR and BF images, respectively. (C-D) Tumor bearing mice received tail vein injections of $2 \mathrm{mg} / \mathrm{kg}$ of the Gem-equivalent dose of various IONPs five times. At the end of the experimental period, tumors were collected and weighed. (C) The mean tumor weights (navy bar) and individual tumor weight distribution of the tumor bearing mice in each group are shown as colored symbols. Values represent mean \pm S.D. of 16 mice from three repeat studies. *Statistically significant difference vs. control, One-Way ANOVA method: $p<0.0001$; Modified t-test: $p<0.0002$. **Statistically significant difference. ATF-IONP-Gem vs. Gem and IO-Gem groups, One-Way ANOVA method: $p<0.05$; Modified t-test: $p<0.05$. (D) Immunohistochemical staining of the cell proliferation marker, Ki-67 in tumor tissue sections. Brown: Ki-67 positive tumor cells. Blue: hematoxylin background staining. Figure and legend reproduced from [6]. 
There are additional studies that focus on X-ray induced photosensitizers, including a study that focused on lanthanide micelles, loaded with the photosensitizer hypericin, that showed promise for X-ray induced nanoparticles involving photosensitizers [144]. Gold has also shown optimal properties for X-ray imaging and X-ray induced ionizing radiation. A study by Huang et al. showed that silica-modified folic acid-functionalized gold nanorods could be used for X-ray or CT imaging, as well as for radiation therapy and photothermal therapy [145]. Another study showed that modified gold nanoparticles exhibited low toxicity in cell and enhanced cancer killing when treated with X-rays compared to cells treated with X-rays without the addition of gold nanoparticles. These gold nanoparticles could then be combined with CT imaging to provide a theranostic platform used for the treatment of cancer [146]. Additionally, there have been studies that focused on the chemotherapeutic release by X-ray irradiation due to increased tissue penetration over light-triggered chemotherapeutic release. One such study focused on doxorubicin conjugated to DNA strands attached to gold nanoparticles, which, upon irradiation, released doxorubicin for chemotherapeutic effects. The properties of gold also allow these particles to be imaged using CT imaging [147]. These are only a few examples of the uses of X-ray irradiation for theranostic treatment methods.

\section{Radiofrequency Triggered Hyperthermia}

Radiofrequency triggered hyperthermia is another popular remotely triggered treatment method. One study conducted by Elsherbini et al. used gold-coated magnetic nanocomposites to induce hyperthermia, both, by laser and radiofrequency irradiation to treat subcutaneous Ehrlich carcinoma in mice. This study observed that more than $50 \%$ of the tumors treated with light or radiofrequency irradiation disappeared completely [96]. Another study focused on doxorubicin loaded $\mathrm{La} 0.7 \mathrm{Sr} 0.3 \mathrm{MnO} 3$ nanoparticles in combination with radiofrequency that triggered drug release as well as hyperthermia effects providing a bimodal treatment method. This method showed effective accumulation of nanoparticles in the cell nucleus, as well as DNA damage, heat shock protein induction, and caspase production, which led to cell death [148]. A study by Wang et al. used gold coated magnetoliposomes for a variety of purposes including radiofrequency triggered release, chemo-hyperthermia therapy, as well as magnetic resonance and $\mathrm{X}$-ray imaging. These gold coated magnetoliposomes showed promising results as drug delivery carriers and optimal imaging properties both in vitro and in vivo [149]. An additional study showed that biodegradable alginate nanoparticles combining radiofrequency triggered hyperthermia and triggered doxorubicin release could be used as an effective combination treatment method. In orthotopic rat liver tumor models, there was enhanced thermal ablation, controlled doxorubicin release, and imaging potential using MRI [150]. X-ray induced ionizing radiation and radiofrequency triggered hyperthermia are only a couple examples of some additional remotely triggered treatments that could be used in theranostic platforms.

\section{Conclusions and Future Directions}

Nanotheranostics has rapidly progressed to include biocompatible and biodegradable, multifunctional and integrated nanoplatforms that encapsulate drugs and diagnostic agents [151-162]. Nanotheranostics may deliver chemo-, radio-, biologic-, immuno- or gene therapies, or some combinations of these agents. An overview of some recent remotely triggered nanotheranostics for the management of cancer are summarized in Table 1. Remotely triggered nanoconstructs show tremendous promise for the treatment of cancer in preclinical models [17, 67, 128, 157, 163-168]. In an ideal world, these nanotheranostics would allow clinicians to diagnose cancer, initiate therapy, and evaluate treatment response while allowing them to track the nanoparticles' pharmacokinetics and release of the payload following application of appropriate external triggers. For these aims to be feasible, such nanotheranostics would have to be designed to either have suitable inherent physicochemical properties or incorporate proper external labels.

For all "smart" nanotheranostic systems, there are several biological impediments that challenge the efficacy of nanoparticle delivery [169]. An ideal nanotheranostic would have to be nonimmunogenic, allow targeted and rapid buildup in tumor tissues, report relevant characteristics of the tissues of interest, deliver effective therapy on demand, monitor response, initiate secondary treatments, and be biocompatible and biodegradable with innocuous derivatives. A new nanoconstruct that is showing promise for future cancer imaging and treatment applications, through detection of macroscopic and microscopic residual disease without a targeting agent in vivo, is the nanostars platform [170]. 
Table 1. Summary of Remotely Triggered Treatments using Nanoparticles for Cancer Applications.

\begin{tabular}{|c|c|c|c|c|c|c|c|}
\hline $\begin{array}{l}\text { Remote } \\
\text { Trigger }\end{array}$ & Therapy & Diagnostic & NP Type & App. Size (nm) & Cancer Type & \begin{tabular}{|l|l} 
In \\
Vivo?
\end{tabular} & Ref \\
\hline \multirow[t]{43}{*}{ Light } & \multirow[t]{17}{*}{ Photodynamic } & \multirow[t]{13}{*}{ Fluorescenc } & Quenched-Activity-Based-Probe (qABP) with photosensitizer & $\mathrm{N} / \mathrm{A}$ & Breast & Yes & 23 \\
\hline & & & Ce6 conjugated carbon dots & $2.5-10$ & Gastric & Yes & 24 \\
\hline & & & PEG functionalized, biodegradable polyacrylamide nanospheres & 44 & Breast & Yes & 25 \\
\hline & & & Ce6 encapsulated and polymer coated upconversion nanoparticles & 30 & Breast, cervical & Yes & 26 \\
\hline & & & $\begin{array}{l}\text { Pyropheophorbide A and c(RGDyK) comodified chitosan wrapped } \\
\text { upconversion nanoparticles }\end{array}$ & $50-53$ & Glioblastoma & No & 27 \\
\hline & & & 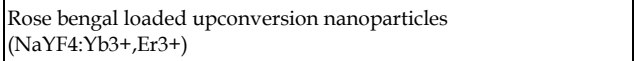 & 20 & Choriocarcinoma & No & 29 \\
\hline & & & Mesotetraphenylporphine loaded peroxalate nanomicelles & $60-120$ & Glioma, colon & No & 30 \\
\hline & & & $\begin{array}{l}\text { Folic acid - targeted, zinc phthalocyanine encapsulated carbon } \\
\text { nanodots }\end{array}$ & 5 & Cervical & Yes & 42 \\
\hline & & & $\begin{array}{l}\text { ZnPc loaded upconverting nanoparticles coated with } \\
\text { folate-modified amphiphilic chitosan (FASOC) }\end{array}$ & 50 & $\begin{array}{l}\text { Adenocarcinoma, breast, } \\
\text { hepatoma, sarcoma }\end{array}$ & Yes & 44 \\
\hline & & & Mesoporous-silica coated upconversion nanoparticles & $100-130$ & Melanoma & Yes & 47 \\
\hline & & & $\mathrm{pH}$ activatable, folic acid-targeted, rubyrin loaded nanoparticles & 150 & Cervical & Yes & 49 \\
\hline & & & $\begin{array}{l}\text { CD-117 targeted indocyanine green loaded calcium phosphosilicate } \\
\text { nanoparticles }\end{array}$ & 40 & Leukemia & Yes & 50 \\
\hline & & & Pthyalocyanine bound Au nanoparticles or liposomes & 5 & Breast & No & 53 \\
\hline & & MRI & $\begin{array}{l}\text { Folic acid - targeted NaGdF4:Yb/ } \\
\text { Tm@Si2@TiO2 nanocomposites }\end{array}$ & 350 & Breast & Yes & 45 \\
\hline & & Fluorescence and MRI & $\begin{array}{l}\text { Hexagonal NaYF4:Y,Er/NaGdF4 core-shell upconverting } \\
\text { nanoparticles }\end{array}$ & 50 & Brain (Glioblastoma) & Yes & 46 \\
\hline & & $\begin{array}{l}\text { Differential interference } \\
\text { contrast, fluorescence }\end{array}$ & Jacalin-PEG functionalized phthalocyanine Au nanoparticles & 4 & Colon, oncofetal & No & 54 \\
\hline & & $\begin{array}{l}\text { Surface enhanced Raman } \\
\text { Spectroscopy (SERS) }\end{array}$ & Protoporphyrin IX conjugated Au nanoparticles & $20-100$ & Breast & No & 55 \\
\hline & \multirow[t]{9}{*}{ Photothermal } & Photoacoustic, optical & TNF- $\alpha$ conjugated, PEG functionalized Au nanospheres & 30 & Mammary breast cancer & Yes & 2 \\
\hline & & Photoacoustic & Plasmonic nanobubbles & 60 & Squamous cell carcinoma & Yes & 16 \\
\hline & & \multirow[t]{3}{*}{ Fluorescence } & Hyaluronic acid receptor-targeted nanographene oxide particles & 250 & Melanoma & Yes & 63 \\
\hline & & & Au nanoparticle-coated Si nanowires & $150 \times 2600$ & Squamous cell carcinoma & No & 65 \\
\hline & & & Chitosan-coated hollow copper sulfide nanoparticles & 85 & Breast & Yes & 69 \\
\hline & & $\begin{array}{l}\text { Fluorescence, MRI, and } \\
\text { photoacoustic }\end{array}$ & $\begin{array}{l}\text { PEG functionalized graphene nanosheets anchored with magnetic } \\
\text { nanoparticles }\end{array}$ & 50 & Breast & Yes & 64 \\
\hline & & $\begin{array}{l}\text { Diffuse optical } \\
\text { tomography }\end{array}$ & Biodegradable indocyanine green loaded PLGA nanoparticles & 250 & Prostrate & No & 97 \\
\hline & & $\begin{array}{l}\text { Surface enhanced Raman } \\
\text { Spectroscopy, } \\
\text { fluorescence }\end{array}$ & Au nano popcorn conjugated single wall carbon nanotubes & 40 & Breast & No & 98 \\
\hline & & Ultrasound & Polypyrrole hollow microspheres & $\mathrm{N} / \mathrm{A}$ & Brain & Yes & 105 \\
\hline & \multirow[t]{2}{*}{\begin{tabular}{|l|} 
Phototriggered \\
chemotherapeuti \\
c release
\end{tabular}} & \multirow[t]{2}{*}{ Fluorescence } & $\begin{array}{l}\text { Photoswitchable hybrid nanoparticles comprised of spiropyran and } \\
\text { lipid-PEG }\end{array}$ & $\begin{array}{l}\text { Change in size } \\
\text { from } 100 \mathrm{~nm} \text { to } \\
50 \mathrm{~nm} \text { upon } \\
\text { light } \\
\text { irradiation }\end{array}$ & Fibrosarcoma & Yes & 78 \\
\hline & & & Targeted doxorubicin conjugate gold nanoparticles & 22 & Leukemia & No & 89 \\
\hline & \multirow{15}{*}{$\begin{array}{l}\text { Photothermal } \\
\text { and } \\
\text { phototriggered }\end{array}$} & & IR820-chitosan conjugates & N/A & Uterine, ovarian & Yes & 7 \\
\hline & & & $\begin{array}{l}\text { Cy3-modified, S6 aptamer conjugated magnetic core-plasmonic } \\
\text { shell nanostars }\end{array}$ & 70 & Breast & No & 8 \\
\hline & & & DOX/ICG-loaded temperature sensitive liposomes & $20-50$ & Adenocarcinoma & Yes & 58 \\
\hline & & & $\begin{array}{l}\text { Doxorubicin loaded Pd nanosheet-covered hollow mesoporous Si } \\
\text { nanoparticles }\end{array}$ & $170-200$ & Hepatoblastoma & No & 76 \\
\hline & & & $\begin{array}{l}\text { Doxorubicin loaded poly(lactic-co-glycolic acid) (PLGA) coated } \\
\text { with NAcHis-TPGS }\end{array}$ & $14-18$ & Prostate & Yes & 87 \\
\hline & & & $\mathrm{pH} /$ thermosensitive copper sulfide nanocapsules & $150-440$ & Melanoma & Yes & 88 \\
\hline & & & Thermosensitive doxorubicin loaded liposomes and Au nanorods & $110-130$ & Glioblastoma & Yes & 92 \\
\hline & & & Au nanorod elastin-like polypeptide matrices & 50 & Prostate & No & 93 \\
\hline & & & $\begin{array}{l}\begin{array}{l}\text { PEG functionalized, doxorubicin loaded graphene oxide } \\
\text { nanoparticles }\end{array} \\
\end{array}$ & 100 & Breast & Yes & 101 \\
\hline & & $\begin{array}{l}\text { Fluorescence, optical } \\
\text { coherence tomography }\end{array}$ & PEG functionalized metal nanoshells & 140 & Colon & Yes & 73 \\
\hline & & $\begin{array}{l}\text { X-ray computed } \\
\text { tomography, infrared } \\
\text { thermal }\end{array}$ & $\begin{array}{l}\text { Polydopamine/human serum albumin/doxorubicin coated } \\
\text { bismuth selenide nanoparticle }\end{array}$ & 110 & Cervical & No & 85 \\
\hline & & $\begin{array}{l}\text { Confocal laser scanning } \\
\text { microscopy }\end{array}$ & Doxorubicin and irinotecan loaded graphene oxide nanocarrier & 200 & Breast & No & 90 \\
\hline & & \multirow{2}{*}{$\begin{array}{l}\text { MRI, infrared thermal, } \\
\text { fluorescence }\end{array}$} & $\mathrm{pH}$ responsive mesoporous silica-coated gold nanorods & 300 & Breast & No & 91 \\
\hline & & & Au capped magnetic core/mesoporous Si shell nanoellipsoids & 380 & Breast & Yes & 95 \\
\hline & & MRI, ultrasound, optical & Antibody C225-conjugated hollow gold nanospheres & 40 & Squamous cell carci & Yes & 94 \\
\hline
\end{tabular}




\begin{tabular}{|c|c|c|c|c|c|c|c|}
\hline & \multirow{2}{*}{$\begin{array}{l}\text { Photodynamic } \\
\text { and } \\
\text { photothermal }\end{array}$} & $\begin{array}{l}\text { Fluorescence, thermal, } \\
\text { and photoacoustic }\end{array}$ & Ce6 encapsulated Au nanovessels & 300 & Breast & Yes & 19 \\
\hline & & Fluorescence & Indocyanine green conjugated Au nanorods and nanoparticles & $13-100$ & Lung & No & 99 \\
\hline & \multirow{4}{*}{$\begin{array}{l}\text { Photodynamic } \\
\text { and } \\
\text { phototriggerred }\end{array}$} & \multirow[t]{3}{*}{ Fluorescence } & $\begin{array}{l}\text { Liposome with BPD in the liquid bilayer and cabozantinib } \\
\text { encapsulated in the core }\end{array}$ & 150 & Pancreatic & Yes & 39 \\
\hline & & & Galactose functionalized mesoporous silica nanoparticles & 245 & Breast, colorectal, pancreatic & No & 51 \\
\hline & & & Carbohydrate functionalized mesoporous silica nanoparticles & N/A & Retinoblastoma & No & 52 \\
\hline & & $\begin{array}{l}\text { Upconversion } \\
\text { luminescence, MRI }\end{array}$ & $\begin{array}{l}\text { Ce6, ZnPc, and methylene blue loaded upconverting nanoparticles } \\
\text { (UCNPs) }\end{array}$ & $20-30$ & Epithelial lung cancer & Yes & 43 \\
\hline & $\begin{array}{l}\text { Photodynamic, } \\
\text { photothermal, } \\
\text { and } \\
\text { phototriggered }\end{array}$ & Fluorescence & Hsp90-inhibitor loaded nanoporphyrin & 22 & Prostrate & No & 40 \\
\hline Electrical & Electro-thermal & Fluorescence & Bacteriobot & $\mathrm{N} / \mathrm{A}$ & Colon & Yes & 122 \\
\hline \multirow[t]{3}{*}{ Magnetic } & \multirow[t]{2}{*}{ Magneto-thermal } & \multirow[t]{2}{*}{ MRI } & $\begin{array}{l}\text { Urokinase plasminogen activator receptor-targeted magnetic iron } \\
\text { oxide NP carrying gemcitabine }\end{array}$ & 70 & Pancreatic & Yes & 6 \\
\hline & & & Folic acid - targeted superparamagnetic nanoparticles & $60-100$ & Myeloma & Yes & 141 \\
\hline & $\begin{array}{l}\text { Magnetoplasmon } \\
\text { ic }\end{array}$ & MRI & $\begin{array}{l}\mathrm{Si} \text { shell encapsulated magnetic } \mathrm{Fe} 3 \mathrm{O} 4 \text { particles conjugated with } \mathrm{Au} \\
\text { nanoparticles; drug loaded and PEG functionalized }\end{array}$ & $130-140$ & Leukemia & No & 165 \\
\hline \multirow[t]{5}{*}{ Sound } & \multirow[t]{5}{*}{ Ultrasound } & Ultrasound & N-cadherin-targeted liposome loaded microbubbles & $1000-10000$ & Melanoma & No & 108 \\
\hline & & \multirow[t]{2}{*}{ MRI } & Gadoteridol loaded liposomes & 150 & Melanoma & Yes & 113 \\
\hline & & & Doxorubicin loaded low temperature sensitive liposomes & N/A & Vx2 carcinoma & Yes & 114 \\
\hline & & $\begin{array}{l}\text { Confocal laser scanning } \\
\text { microscopy, ultrasound }\end{array}$ & Biodegradable poly(methacrylic acid) based nanocapsules & 300 & Cervical & Yes & 115 \\
\hline & & MRI, ultrasound & $\begin{array}{l}\text { Superparamagnetic iron oxide and drug complex-embedded } \\
\text { acoustic droplets }\end{array}$ & $1200-1500$ & Glioma & Yes & 116 \\
\hline \multirow[t]{3}{*}{ X-Ray } & \multirow{2}{*}{$\begin{array}{l}\text { X-ray triggered } \\
\text { PDT }\end{array}$} & Fluorescence & CeF3 nanoparticles conjugated with the photosensitizer verteporfin & 10 & Leukemia & Yes & 143 \\
\hline & & MRI & Lanthanide-based micelles integrating hypericin & 5 & HeLa cervical cancer & Yes & 144 \\
\hline & $\begin{array}{l}\text { X-Ray radiation } \\
\text { therapy }\end{array}$ & X-ray, CT & Folic acid-conjugated Silica-modified gold nanorods & $\begin{array}{l}50 \text { (length) } \times 18 \\
\text { (width) }\end{array}$ & Gastric & No & 145 \\
\hline $\begin{array}{l}\text { Radiofreq } \\
\text { uency and } \\
\text { light }\end{array}$ & $\begin{array}{l}\text { Radiofrequency } \\
\text { and light } \\
\text { triggered } \\
\text { hyperthermia }\end{array}$ & MRI & Au-coated $\mathrm{Fe} 3 \mathrm{O} 4$ magnetic nanocomposites & 55 & Ehrlich carcinoma & Yes & 96 \\
\hline \multirow[t]{3}{*}{$\begin{array}{l}\text { Radiofreq } \\
\text { uency }\end{array}$} & \multirow{3}{*}{$\begin{array}{l}\text { Radiofrequency } \\
\text { triggered } \\
\text { hyperthermia } \\
\text { and drug release }\end{array}$} & Fluorescence & $\begin{array}{l}\text { Chitosan coated La0.7Sr } 0.3 \mathrm{MnO} 3 \text { nanoparticles loaded with } \\
\text { doxorubicin }\end{array}$ & $50-300$ & Breast & Yes & 148 \\
\hline & & X-ray, MRI & $\begin{array}{l}\text { Doxorubicin-loaded thermosensitive magnetoliposomes } \\
\text { conjugated with Au nanospheres and PEG }\end{array}$ & 200 & Liver & No & 149 \\
\hline & & MRI & Stannous-doped alginate NPs coloaded with doxorubicin & $100-200$ & Liver & No & 150 \\
\hline
\end{tabular}

An important challenge in the translation of remotely triggered theranostics is that some of the current externally activated systems have very low efficacies with significant toxicities. For example, phototriggered systems have low photothermal conversion, aka or reactive oxygen species generation efficiency of photosensitizers, with low tumor-to-normal cell ratios during biodistribution studies. An additional issue is the limited tissue dissemination of the radiation that is required for remote initiation that impedes the treatment of deep tissue cancers. Longer wavelength radiation sources can penetrate deeper into the tissue, but the selection of the source is governed by the choice of the photosensitizers. Toxicity is also a major challenge for the clinical translation of inorganic nanoparticle-based remotely triggered nanosystems that may present acute and chronic toxicities. These challenges must be addressed prior to designing a remotely activated theranostic nanosystem.

It is well established that the size, morphology, stiffness, surface charge, and surface chemistry of nanoparticles can tremendously impact their behavior and performance in vivo. There are a variety of ways to achieve accurate control and scalability in theranostic nanomedicine. A number of remote triggers can be used to achieve precise control over the nanotheranostic systems reviewed here [171,172]. There is no "perfect" trigger mechanism that may be universally applied to achieve superlative results in every application. Instead, the choice of trigger should be based on the final application and the resources available. Light has a perceived penetration depth problem that some of the other triggers reviewed here do not exhibit. The recent developments in fiber optic probes and endoscopy may help overcome some of these issues for phototriggers. Certain triggers like $\mathrm{X}$-ray may increase the radiation burden, which should be considered [172].

On account of the inherent intricacy of remotely triggered nanotheranostics by design, the clinical translation of these platforms is not trivial. There are several challenges that need to be addressed in order to generate successful clinical applications [173-182]. In magnetic hyperthermia, these issues include limited light penetration depth, and in some cases, 
insufficient heating. Research has been conducted on upconverting nanoparticles to try to increase tissue penetration through sequential absorption by converting NIR light to shorter wavelengths. It has also been suggested to use light irradiation to destroy small tumors after the primary tumors are surgically removed. For successful use of magnetic hyperthermia, materials with higher magnetic heating ability must be used so that there is enough heat generated to destroy the tumor.

Not only are there issues with the treatment methods, but there are also challenges with the behaviors of the nanoparticles moving from in vivo to clinical trials in regards to how the nanoparticles act in the human body (i.e. biodistribution, toxicity, and degradation). Although a nanostructure may prove effective in vivo, it may not necessarily be suitable for clinical trials. These are just some of the challenges that need to be addressed if remotely triggered theranostic treatments are to be used for clinical applications [182, 183]. The design procedures therefore need to be iterative, and researchers need to adapt and evolve their nanoplatform designs based on the vital lessons learned from other researchers who may or may not have been successful with their remotely triggered nanotheranostic system.

Robust and reproducible procedures that enable relatively easy and cost effective scale-up and manufacturing of nanotheranostic platforms are essential for the clinical translation and the prolonged success of these "smart" systems. Findings of physiological reactions to such novel, integrated nanosystems need to contemplate many factors, including dosage (effective levels for diagnostic vs. therapeutic efficacy), exposure levels, systemic buildup, elimination kinetics, tissue and organ distributions, and the physiology of potential patients. It is imperative to comprehend the potential and perceived and acute as well as chronic toxic side-effects before they can be tested in humans to diagnose and treat cancers [173-182, 184-190].

The compilation of work described here establishes that multiplexed "smart" nanosystems that integrate diagnostic and therapeutic agents are capable of triggering drug release at cancer sites when activated by external stimuli and can be extremely potent in understanding cancer through real-time observation of drug delivery, release, and activity. Although more investigation and testing is needed before remotely triggered theranostics can be realized in the clinic, current research suggests that such nanotheranostics may transform the diagnosis and treatment of several diseases, and help fulfill the potential of tailored, individualized nanomedicine $[17,67,128,157,163-168,173-182,184-190]$.

\section{Acknowledgements}

The authors acknowledge funding from the National Cancer Institute, a part of the National Institutes of Health (Award \# R00CA153948).

\section{Conflict of Interest}

The authors have declared that no conflict of interest exists.

\section{References}

1. Siegel RL, Miller KD, Jemal A. Cancer Statistics, 2016. CA: Cancer J Clin. 2016; 66(1): 7-30.

2. Shao J, Griffin RJ, Galanzha EI, et al. Photothermal nanodrugs: potential of TNF-gold nanospheres for cancer theranostics. Sci Rep. 2013; 3: 1293.

3. Lo ST, Kumar A, Hsieh JT, Sun X. Dendrimer nanoscaffolds for potential theranostics of prostate cancer with a focus on radiochemistry. Mol Pharm. 2013; 10: 793-812.

4. Brakmane G, Madani SY, Seifalian A. Cancer Antibody Enhanced Real Time Imaging Cell Probes - A Novel Theranostic Tool using Polymer Linked Carbon Nanotubes and Quantum Dots. Anticancer Agents Med Chem. 2013.

5. Frigerio B, et al. A single-chain fragment against prostate specific membrane antigen as a tool to build theranostic reagents for prostate cancer. Eur J Cancer. 2013.

6. Lee GY, et al. Theranostic nanoparticles with controlled release of gemcitabine for targeted therapy and MRI of pancreatic cancer. ACS Nano. 2013; 7: 2078-2089.

7. Srinivasan S, Manchanda R, Fernandez-Fernandez A, Lei T, McGoron AJ. Near-infrared fluorescing IR820-chitosan conjugate for multifunctional cancer theranostic applications. J Photochem Photobiol B. 2013; 119: 52-59.

8. Fan Z, Senapati D, Singh AK, Ray PC. Theranostic magnetic core-plasmonic shell star shape nanoparticle for the isolation of targeted rare tumor cells from whole blood, fluorescence imaging, and photothermal destruction of cancer. Mol Pharm. 2013; 10: 857-866.

9. Muthu MS, Feng SS. Theranostic liposomes for cancer diagnosis and treatment: current development and pre-clinical success. Expert Opin Drug Deliv. 2013; 10: 151-155.

10. Ikeda T, Mitsuyama S. New challenges in the field of breast cancer therapy-do we need surgery for the patients with breast cancer? Breast Cancer. 2007; 14: 37-38.

11. Meurisse M, Defechereux T, Meurisse N, Bataille Y, Hamoir E. New challenges in the treatment of early breast cancer or surgery for early breast cancer ... can less be more? Acta Chir Belg. 2002; 102: 97-109.

12. Giacomantonio CA, Temple WJ. Quality of cancer surgery: challenges and controversies. Surg Oncol Clin N Am. 2000; 9: 51-60, vii.

13. Funkhouser J. Reinventing pharma: The theranostic revolution. Current Drug Discovery. 2002; 2: 17-19.

14. Lukianova-Hleb E, Hanna EY, Hafner JH, Lapotko DO. Tunable plasmonic nanobubbles for cell theranostics. Nanotechnology. 2010; 21(8): 085102.

15. Lukianova-Hleb $E$, Ren $X$, Sawant $R R$, et al. On-demand intracellular amplification of chemoradiation with cancer-specific plasmonic nanobubbles. Nature Medicine. 2014; 20(7): 778-784.

16. Lukianova-Hleb EY, Kim YS, Belatsarkouski I, et al. Intraoperative Diagnostics and Elimination of Residual Microtumours with Plasmonic Nanobubbles. Nature Nanotechnology. 2016; 11(6): 525-32.

17. Lukianova-Hleb EY, Oginsky AO, Samaniego AP, et al. Tunable plasmonic nanoprobes for theranostics of prostate cancer. Theranostics. 2011; 1: 3-17.

18. Lukianova-Hleb EY, Ren X, Zasadzinski JA, et al. Plasmonic nanobubbles enhance efficacy and selectivity of chemotherapy against drug-resistant cancer cells. Adv Mater. 2012; 24: 3831-3837.

19. Lin J, et al. Photosensitizer-Loaded Gold Vesicles with Strong Plasmonic Coupling Effect for Imaging-Guided Photothermal/Photodynamic Therapy. ACS Nano. 2013; 7(6): 5320-5329.

20. Feng $\mathrm{X}$, et al. A novel folic acid-conjugated $\mathrm{TiO} 2-\mathrm{SiO} 2$ photosensitizer for cancer targeting in photodynamic therapy. Colloids and Surfaces B: Biointerfaces. 2015; 125: 197-205.

21. Park BJ, et al. Photodynamic Anticancer Activities of Multifunctional Cobalt Ferrite Nanoparticles in Various Cancer Cells. Journal of Biomedical Nanotechnology. 2015; 11: 226-235.

22. Chen $\mathrm{WH}$, et al. Nanoparticle delivery of HIF1a siRNA combined with photodynamic therapy as a potential treatment strategy for head-and-neck cancer. Cancer Letters. 2015; 359: 65-74.

23. Ben-Nun Y, et al. Photodynamic Quenched Cathepsin Activity Based Probes for Cancer Detection and Macrophage Targeted Therapy. Theranostics. 2015; 5(8): 847-862.

24. Huang $\mathrm{P}$, et al. Light-Triggered Theranostics Based on Photosensitizer-Conjugated Carbon Dots for Simultaneous Enhanced-Fluorescence Imaging and Photodynamic Therapy. Adv Mater. 2012; 24(37): 5104-5110. 
25. Wang S, et al. Multifunctional Biodegradable Polyacrylamide Nanocarriers for Cancer Theranostics - A "See and Treat" Strategy. ACS Nano. 2012.

26. Wang C, Tao H, Cheng L, Liu Z. Near-infrared light induced in vivo photodynamic therapy of cancer based on upconversion nanoparticles. Biomaterials. 2011; 32: 6145-6154.

27. Zhou A, Wei Y, Wu B, Chen Q, Xing D. Pyropheophorbide A and c(RGDyK) Comodified Chitosan-Wrapped Upconversion Nanoparticle for Targeted Near-Infrared Photodynamic Therapy. Mol Pharm. 2012; 9: 1580-1589.

28. Zhao $\mathrm{Z}$, et al. Multifunctional core-shell upconverting nanoparticles for imaging and photodynamic therapy of liver cancer cells. Chem Asian J. 2012; 7: 830-837.

29. Liu K, et al. Covalently Assembled NIR Nanoplatform for Simultaneous Fluorescence Imaging and Photodynamic Therapy of Cancer Cells. ACS Nano. 2012.

30. Chen R, Zhang L, Gao J, et al. Chemiluminescent nanomicelles for imaging hydrogen peroxide and self-therapy in photodynamic therapy. J Biomed Biotechnol. 2011; 679492.

31. Ferrara KW. Driving delivery vehicles with ultrasound. Adv Drug Deliv Rev. 2008; 60: 1097-1102.

32. Deckers $\mathrm{R}$, Moonen CT. Ultrasound triggered, image guided, local drug delivery. J Control Release. 2010; 148: 25-33.

33. Stoffels I, et al. Metastatic Status of Sentinel Lymph Nodes in Melanoma Determined Noninvasively with Multispectral Optoacoustic Imaging. Science Translational Medicine. 2015; 7(317).

34. Simon DT, et al. Organic electronics for precise delivery of neurotransmitters to modulate mammalian sensory function. Nat Mater. 2009; 8: 742-746.

35. Shenoi MM, et al. Nanoparticle Delivered Vascular Disrupting Agents (VDAs): Use of TNF-alpha conjugated Gold Nanoparticles for Multimodal Cancer Therapy. Mol Pharm. 2013; 10(5): 1683-1694.

36. Rai $\mathrm{P}$, et al. Development and Applications of Photo-triggered Theranostic Agents. Adv Drug Deliv Rev. 2010; 62(11): 1094-1124

37. [Internet] The U.S. National Institutes of Health. ClinicalTrials.gov. Phase 3 Study of ThermoDox With Radiofrequency Ablation (RFA) in Treatment of $\begin{array}{llll}\text { Hepatocellular } & \text { Carcinoma } & \text { (HCC). } & \text { February } \\ \end{array}$ https://clinicaltrials.gov/ct2/show/NCT00617981

38. Dolmans DE, Fukumura D, Jain RK. Photodynamic therapy for cancer. Nat Rev Cancer. 2003; 3: 380-387.

39. Spring BQ, et al. A Photoactivable Multi-inhibitor Nanoliposome for Tumour Control and Simultaneous Inhibition of Treatment Escape Pathways. Nature Nanotechnology. 2016; 11(4): 378-87.

40. Lin T, et al. HSP90 Inhibitor Encapsulated Photo-Theranostic Nanoparticles for Synergistic Combination Cancer Therapy. Theranostics. 2016; 6(9): 1324-1335.

41. Uppal A, Jain B, Gupta PK, Das K. Photodynamic action of Rose Bengal silica nanoparticle complex on breast and oral cancer cell lines. Photochem Photobiol. 2011; 87: 1146-1151.

42. Choi Y, et al. Highly Biocompatible Carbon Nanodots for Simultaneous Bioimaging and Targeted Photodynamic Therapy In Vitro and In Vivo. Advanced Functional Materials. 2014; 24(37): 5781-5789.

43. Tian G, et al. Red-Emitting Upconverting Nanoparticles for Photodynamic Therapy in Cancer Cells Under Near-Infrared Excitation. Small. 2013; 9(11): 1929-1938.

44. Cui S, et al. In Vivo Targeted Deep-Tissue Photodynamic Therapy Based on Near-Infrared Light Triggered Upconversion Nanoconstruct. ACS Nano. 2013; 7(1): 676-688.

45. Zhang L, et al. Inorganic photosensitizer coupled Gd-based upconversion luminescent nanocomposites for in vivo magnetic resonance imaging and near-infrared-responsive photodynamic therapy in cancers. Biomaterials. 2015; 44: 82-90.

46. Park YI, et al. Theranostic Probe Based on Lanthanide-Doped Nanoparticles for Simultaneous In Vivo Dual-Modal Imaging and Photodynamic Therapy. Advanced Materials. 2012; 24: 5755-5761.

47. Idris $\mathrm{NM}$, et al. In vivo photodynamic therapy using upconversion nanoparticles as remote-controlled nanotransducers. Nature Medicine. 2012; 18(10): 1580-1585.

48. Shi J, et al. The application of hyaluronic acid-derivatized carbon nanotubes in hematoporphyrin monomethyl ether-based photodynamic therapy for in vivo and in vitro cancer treatment. International Journal of Nanomedicine. 2013; 8: 2361-2373.

49. Tian J, et al. Cell-Specific and pH-Activatable Rubyrin-Loaded Nanoparticles for Highly Selective Near-Infrared Photodynamic Therapy against Cancer. Journal of the American Chemical Society. 2013; 135: 18850-18858.

50. Barth BM, et al. Targeted indocyanine-green-loaded calcium phosphosilicate nanoparticles for in vivo photodynamic therapy of leukemia. ACS Nano. 2011; 5: 5325-5337.

51. Gary-Bobo M, Hocine O, Brevet D, et al. Cancer therapy improvement with mesoporous silica nanoparticles combining targeting, drug delivery and PDT. Int J Pharm. 2012; 423: 509-515.

52. Gary-Bobo M, Mir Y, Rouxel C, et al. Multifunctionalized mesoporous silica nanoparticles for the in vitro treatment of retinoblastoma: Drug delivery, one and two-photon photodynamic therapy. Int J Pharm. 2012; 432: 99-104.

53. Nombona N, Maduray K, Antunes E, Karsten A, Nyokong T. Synthesis of phthalocyanine conjugates with gold nanoparticles and liposomes for photodynamic therapy. J Photochem Photobiol B. 2012; 107: 35-44.
54. Obaid G, Chambrier I, Cook MJ, Russell DA. Targeting the Oncofetal Thomsen-Friedenreich Disaccharide Using Jacalin-PEG Phthalocyanine Gold Nanoparticles for Photodynamic Cancer Therapy. Angew Chem Int Ed Engl. 2012.

55. Khaing Oo MK, Yang $\mathrm{Y}, \mathrm{Hu} \mathrm{Y}$, et al. Gold nanoparticle-enhanced and size-dependent generation of reactive oxygen species from protoporphyrin IX. ACS Nano. 2012; 6: 1939-1947.

56. Lindner U, Weersink RA, Haider MA, et al. Image guided photothermal focal therapy for localized prostate cancer: phase I trial. J Urol. 2009; 182: 1371-1377.

57. Jacques SL. Laser-tissue interactions: Photochemical, photothermal, and photomechanical. Surg Clin North Am. 1992; 72: 531-558.

58. Zhao P, et al. NIR-driven Smart Theranostic Nanomedicine for On-demand Drug Release and Synergistic Antitumor Therapy. Scientific Reports. 2015; 5: 14258.

59. Koonce NA, et al. Combination of Gold Nanoparticle-Conjugated Tumor Necrosis Factor-a and Radiation Therapy Results in a Synergistic Antitumor Response in Murine Carcinoma Models. Int J Radiation Oncol Biol Phys. 2015; 93(3): 588-596.

60. Iltisa I, et al. In vivo detection of the effects of preconditioning on LNCaP tumors by a TNF-a nanoparticle construct using MRI. NMR Biomed. 2014; 27: 1063-1069.

61. Somasuntharam I, et al. Knockdown of TNF-a by DNAzyme Gold Nanoparticles as an Anti-inflammatory Therapy for Myocardial Infarction. Biomaterials. 2016; 83: 12-22.

62. Shao J, et al. Photothermal nanodrugs: potential of TNF-gold nanospheres for cancer theranostics. Sci. Rep. 2013; 3:1293

63. Jung HS, et al. Nanographene Oxide Hyaluronic Acid Conjugate for Photothermal Ablation Therapy of Skin Cancer. ACS Nano. 2014; 8(1): 260-268.

64. Yang $\mathrm{K}, \mathrm{Hu} \mathrm{L}, \mathrm{Ma} \mathrm{X}$, et al. Multimodal imaging guided photothermal therapy using functionalized graphene nanosheets anchored with magnetic nanoparticles. Adv Mater. 2012; 24: 1868-1872.

65. Su Y, Wei X, Peng F, et al. Gold nanoparticles-decorated silicon nanowires as highly efficient near-infrared hyperthermia agents for cancer cells destruction. Nano Lett. 2012; 12: 1845-1850.

66. Zhang $\mathrm{H}$, Tian M, Li E, et al. Molecular imaging-guided theranostics and personalized medicine. J Biomed Biotechnol. 2012; 747416.

67. Singh SP. Multifunctional magnetic quantum dots for cancer theranostics. J Biomed Nanotechnol. 2011; 7: 95-97.

68. McCoy CP, Brady C, Cowley JF, et al. Triggered drug delivery from biomaterials. Expert Opin Drug Deliv. 2010; 7: 605-616.

69. Guo L, et al. Combinatorial Photothermal and Immuno Cancer Therapy Using Chitosan-Coated Hollow Copper Sulfide Nanoparticles. ACS Nano. 2014; 8(6): 5670-5681.

70. Gutwein LG, Singh AK, Hahn MA, et al. Fractionated photothermal antitumor therapy with multidye nanoparticles. Int J Nanomedicine. 2012; 7: 351-357.

71. Wang X, Wang C, Cheng L, et al. Noble metal coated single-walled carbon nanotubes for applications in surface enhanced Raman scattering imaging and photothermal therapy. J Am Chem Soc. 2012; 134: 7414-7422.

72. Hong $\mathrm{C}$, Lee J, Zheng $\mathrm{H}$, et al. Porous silicon nanoparticles for cancer photothermotherapy. Nanoscale Res Lett. 2011; 6: 321.

73. Gobin AM, Lee MH, Halas NJ, et al. Near-infrared resonant nanoshells for combined optical imaging and photothermal cancer therapy. Nano Lett. 2007; 7: 1929-1934.

74. Boca SC, Potara M, Gabudean AM, et al. Chitosan-coated triangular silver nanoparticles as a novel class of biocompatible, highly effective photothermal transducers for in vitro cancer cell therapy. Cancer Lett. 2011; 311: 131-140.

75. Tang S, et al. Sub-10-nm Pd Nanosheets with Renal Clearance for Efficient Near-Infrared Photothermal Cancer Therapy. Small. 2014; 10(15): 3139-3144.

76. Fang W, et al. Pd Nanosheet-Covered Hollow Mesoporous Silica Nanoparticles as a Platform for the Chemo-Photothermal Treatment of Cancer Cells. Small. 2012; 8(24): 3816-3822.

77. Alvarez-Lorenzo C, Bromberg L, Concheiro A. Light-sensitive intelligent drug delivery systems. Photochem Photobiol. 2009; 85: 848-860.

78. Tong $\mathrm{R}$, et al. Photoswitchable Nanoparticles for in Vivo Cancer Chemotherapy. Proceedings of the National Academy of Sciences. 2013; 110(47): 19048-9053.

79. Liu Y, et al. Fabrication of Multifunctional SiO2@GN-Serum Composites for Chemo-Photothermal Synergistic Therapy. ACS Appl. Mater. Interfaces. 2015; 7: 112-121.

80. Luo D, Carter KA, Miranda D, Lovell JF. Chemophototherapy: An Emerging Treatment Option for Solid Tumors. Adv Sci. 2016.

81. Luo D, et al. Doxorubicin encapsulated in stealth liposomes conferred with light-triggered drug release. Biomaterials. 2016; 75: 193-202.

82. Zhong Y, et al. Gold Nanorod-Cored Biodegradable Micelles as a Robust and Remotely Controllable Doxorubicin Release System for Potent Inhibition of Drug-Sensitive and -Resistant Cancer Cells. Biomacromolecules. 2013; 14: 2411-2419.

83. Jin $Q$, et al. Biocompatible Drug Delivery System for Photo-Triggered Controlled Release of 5-Fluorouracil. Biomacromolecules. 2011; 12: 3684-3691.

84. Lv C, et al. Photodegradable Polyurethane Self-Assembled Nanoparticles for Photocontrollable Release. Langmuir. 2012; 28: 9387-9394.

85. Li Z, et al. Multifunctional Bismuth Selenide Nanocomposites for Antitumor Thermo-Chemotherapy and Imaging. ACS Nano. 2016; 10 (1): 984-997.

86. Voliani $\mathrm{V}$, et al. Cancer phototherapy in living cells by multiphoton release of doxorubicin from gold nanospheres. J. Mater. Chem. B. 2013; 1. 
87. Hung CC, et al. Active Tumor Permeation and Uptake of Surface Charge-Switchable Theranostic Nanoparticles for Imaging-Guided Photothermal/Chemo Combinatorial Therapy. Theranostics. 2016; 6(3): 302-317.

88. Huang S, et al. Smart Cu1.75S Nanocapsules with High and Stable Photothermal Efficiency for NIR Photo-Triggered Drug Release. Nano Research. 2015; 8(12): 4038-4047.

89. Luo YL, et al. Release of Photoactivatable Drugs from Plasmonic Nanoparticles for Targeted Cancer Therapy. ACS Nano. 2011; 5(10): 7796-7804.

90. Tran TH, et al. Development of a Graphene Oxide Nanocarrier for Dual-Drug Chemo-phototherapy to Overcome Drug Resistance in Cancer. ACS Appl. Mater. Interfaces. 2015; 7: 28647-28655.

91. Zhang Z, Wang L, Wang J, et al. Mesoporous silica-coated gold nanorods as a light-mediated multifunctional theranostic platform for cancer treatment. Adv Mater. 2012; 24: 1418-1423.

92. Agarwal A, Mackey MA, El-Sayed MA, Bellamkonda RV. Remote triggered release of doxorubicin in tumors by synergistic application of thermosensitive liposomes and gold nanorods. ACS Nano. 2011; 5: 4919-4926.

93. Huang HC, Yang $\mathrm{Y}$, Nanda A, et al. Synergistic administration of photothermal therapy and chemotherapy to cancer cells using polypeptide-based degradable plasmonic matrices. Nanomedicine (Lond). 2011; 6: 459-473.

94. Melancon MP, Elliott AM, Shetty A, et al. Near-infrared light modulated photothermal effect increases vascular perfusion and enhances polymeric drug delivery. J Control Release. 2011; 156: 265-272.

95. Ma M, Chen H, Chen Y, et al. Au capped magnetic core/mesoporous silica shell nanoparticles for combined photothermo-/chemo-therapy and multimodal imaging. Biomaterials. 2012; 33: 989-998.

96. Elsherbini AA, Saber M, Aggag M, et al. Laser and radiofrequency-induced hyperthermia treatment via gold-coated magnetic nanocomposites. Int J Nanomedicine. 2011; 6: 2155-2165.

97. Patel RH, Wadajkar AS, Patel NL, et al. Multifunctionality of indocyanine green-loaded biodegradable nanoparticles for enhanced optical imaging and hyperthermia intervention of cancer. J Biomed Opt. 2012; 17: 046003.

98. Beqa L, Fan Z, Singh AK, et al. Gold nano-popcorn attached SWCNT hybrid nanomaterial for targeted diagnosis and photothermal therapy of human breast cancer cells. ACS Appl Mater Interfaces. 2011; 3: 3316-3324.

99. Kuo WS, Chang YT, Cho KC, et al. Gold nanomaterials conjugated with indocyanine green for dual-modality photodynamic and photothermal therapy. Biomaterials. 2012; 33: 3270-3278.

100. Botella P, Ortega I, Quesada M, et al. Multifunctional hybrid materials for combined photo and chemotherapy of cancer. Dalton Trans. 2012.

101. Zhang W, Guo Z, Huang D, et al. Synergistic effect of chemo-photothermal therapy using PEGylated graphene oxide. Biomaterials. 2011; 32: 8555-8561.

102. Qin G, Li Z, Xia R, et al. Partially polymerized liposomes: stable against leakage yet capable of instantaneous release for remote controlled drug delivery. Nanotechnology. 2011; 22: 155605.

103. Kost J, Leong K, Langer R. Ultrasound-enhanced polymer degradation and release of incorporated substances. Proc Natl Acad Sci U S A. 1989; 86: 7663-7666.

104. Zhou Y, Wang D, Zhang Y, Chitgupi U, et al. A phodphorous phthalocyanine formulation with intense absorbance at $1000 \mathrm{~nm}$ for deep optical imaging. Theranostics. 2016; 6(5): 688-697.

105. Zha Z, et al. Polypyrrole Hollow Microspheres as Echogenic Photothermal Agent for Ultrasound Imaging Guided Tumor Ablation. Scientific Reports. 2013; 3: 2360.

106. Gao Y, et al. Controlled nanoparticle release from stable magnetic microbubble oscillations. NPG Asia Materials. 2016; 8: 260.

107. Kang ST, et al. Internal polymer scaffolding in lipid-coated microbubbles for control of inertial cavitation in ultrasound theranostics. J. Mater. Chem. B. 2015; 3: 5938

108. Geers B, et al. Targeted Liposome-Loaded Microbubbles for Cell-Specific Ultrasound-Triggered Drug Delivery. Small. 2013; 9(23): 4027-4035.

109. Kiessling F, et al. Ultrasound Microbubbles for Molecular Diagnosis, Therapy, and Theranostics. J Nucl Med. 2012; 53: 345-348.

110. Yudina A, et al. Ultrasound-mediated intracellular drug delivery using microbubbles and temperature-sensitive liposomes. Journal of Controlled Release. 2011; 155: 442-448.

111. Staruch R, et al. Localised drug release using MRI-controlled focused ultrasound Hyperthermia. International Journal of Hyperthermia. 2011; 27(2): 156-171.

112. Ibsen S, et al. A novel nested liposome drug delivery vehicle capable of ultrasound triggered release of its payload. Journal of Controlled Release. 2011; 155: 358-366.

113. Rizzitelli S, et al. In vivo MRI visualization of release from liposomes triggered by local application of pulsed low-intensity non-focused ultrasound. Nanomedicine: Nanotechnology, Biology, and Medicine. 2014; 10: 901-904.

114. Ranjan A, Jacobs GC, Woods DL, et al. Image-guided drug delivery with magnetic resonance guided high intensity focused ultrasound and temperature sensitive liposomes in a rabbit Vx2 tumor model. J Control Release. 2012; 158: 487-494

115. Yang P, et al. Stimuli-responsive biodegradable poly(methacrylic acid) based nanocapsules for ultrasound traced and triggered drug delivery system. Biomaterials. 2014; 35: 2079-2088.
116. Wang $\mathrm{CH}$, et al. Superparamagnetic iron oxide and drug complex-embedded acoustic droplets for ultrasound targeted theranosis. Biomaterials. 2013; 34: $1852-1861$

117. Lee SF, et al. Ultrasound, $\mathrm{pH}$, and Magnetically Responsive Crown-Ether-Coated Core/Shell Nanoparticles as Drug Encapsulation and Release Systems. ACS Appl. Mater. Interfaces. 2013; 5: 1566-1574.

118. Paris JL, et al. Polymer-Grafted Mesoporous Silica Nanoparticles as Ultrasound-Responsive Drug Carriers. ACS Nano. 2013; 9(11): 11023-11033.

119. Min HS, et al. Echogenic Glycol Chitosan Nanoparticles for Ultrasound-Triggered Cancer Theranostics. Theranostics. 2015; 5(12): 1402-1418.

120. Moscicka-Studzinska A, Czarnecka K, Ciach T. Electrically enhanced and controlled drug delivery through buccal mucosa. Acta Pol Pharm. 2008; 65: 767-769.

121. Svirskis D, Travas-Sejdic J, Rodgers A, Garg S. Electrochemically controlled drug delivery based on intrinsically conducting polymers. J Control Release. 2010; 146: 6-15.

122. Park SJ, et al. New Paradigm for Tumor Theranostic Methodology Using Bacteria-based Microrobot. Scientific Reports. 2013; 3.

123. Ge J, Neofytou E, Cahill TJ, et al. Drug release from electric-field-responsive nanoparticles. ACS Nano. 2012; 6: 227-233.

124. Katz E, et al. Substance Release Triggered by Biomolecular Signals in Bioelectronic Systems. J. Phys. Chem. Lett. 2015; 6(8): 1340-1347.

125. Hondroulis E, et al. Immuno Nanoparticles Integrated Electrical Control of Targeted Cancer Cell Development Using Whole Cell Bioelectronic Device. Theranostics. 2014; 4(9): 919-930.

126. Jin Z, et al. Electrochemically Controlled Drug-Mimicking Protein Release from Iron-Alginate Thin-Films Associated with an Electrode. ACS Appl. Mater. Interfaces. 2012; 4: 466-475.

127. Liu Y, et al. An electric-field responsive microsystem for controllable miniaturised drug delivery applications. Sens. Actuators B: Chem. 2012; 175: 100-105.

128. Timko BP, Kohane DS. External electric fields trigger drug release from new hydrogel formulation. Nanomedicine (Lond). 2012; 7: 316

129. Balogh D, Tel-Vered R, Freeman R, Willner I. Photochemically and electrochemically triggered Au nanoparticles "sponges". J Am Chem Soc. 2011; 133: 6533-6536.

130. Lee SK, et al. Nanowire substrate-based laser scanning cytometry for quantitation of circulating tumor cells. Nano Lett. 2012; 12: 2967-2704.

131. Venkatanarayanan A, Keyes TE, Forster RJ. Label-free impedance detection of cancer cells. Anal Chem. 2013; 85: 2216-2222.

132. Edelman ER, Brown L, Taylor J, Langer R. In vitro and in vivo kinetics of regulated drug release from polymer matrices by oscillating magnetic fields. J Biomed Mater Res. 1987; 21: 339-353.

133. Edelman ER, Langer R. Optimization of release from magnetically controlled polymeric drug release devices. Biomaterials. 1993; 14: 621-626.

134. Brazel CS. Magnetothermally-responsive nanomaterials: combining magnetic nanostructures and thermally-sensitive polymers for triggered drug release. Pharm Res. 2009; 26: 644-656.

135. Hoare T, Timko BP, Santamaria J, et al. Magnetically triggered nanocomposite membranes: a versatile platform for triggered drug release. Nano Lett. 2011; 11: 1395-1400.

136. Melancon MP, Stafford RJ, Li C. Challenges to effective cancer nanotheranostics. J Control Release. 2012; 164: 177-182.

137. Ghosh D, et al. M13-templated magnetic nanoparticles for targeted in vivo imaging of prostate cancer. Nature Nanotechnology. 2012; 7: 677-682.

138. Chen R, et al. Wireless magnetothermal deep brain stimulation. Science. 2015; 347(6229): 1477-1480.

139. Oliveira $\mathrm{H}$, et al. Magnetic field triggered drug release from polymersomes for cancer therapeutics. Journal of Controlled Release. 2013; 169: 165-170.

140. Reimhult E. Nanoparticle-triggered release from lipid membrane vesicles. New Biotechnol. 2015; 32(6): 665-672.

141. Hayashi K, et al. Superparamagnetic Nanoparticle Clusters for Cancer Theranostics Combining Magnetic Resonance Imaging and Hyperthermia Treatment. Theranostics. 2013; 3(6): 366-376.

142. Akhavan $\mathrm{O}$, et al. Zinc ferrite spinel-graphene in magneto-photothermal therapy of cancer. J. Mater. Chem. B. 2014, 2: 3306.

143. Clement S, Deng W, Camilleri E, Wilson BC, Goldys EM. X-ray induced singlet oxygen generation by nanoparticle-photosensitizer conjugates for photodynamic therapy: determination of singlet oxygen quantum yield. Sci Rep. 2016; 6(19954).

144. Kascakova S, et al. X-Ray induced RadioPhotoDynamic Therapy (RPDT) using lanthanide micelles: beyond depth limitations. Nano Res. 2015.

145. Huang $\mathrm{P}$, et al. Folic acid-conjugated Silica-modified gold nanorods for $\mathrm{X}$-ray/CT imaging-guided dual-mode radiation and photo-thermal therapy. Biomaterials. 2011; 32(36): 9796-9809.

146. Kong T, et al. Enhancement of Radiation Cytotoxicity in Breast-Cancer Cells by Localized Attachment of Gold Nanoparticles. Small. 2008; 4(9): 1537-1543.

147. Starkewolf B, Miyachi L, Wong J, Guo T. X-ray triggered release of doxorubicin from nanoparticle drug carriers for cancer therapy. Chem Commun. 2013; 49(25): 2545-2547.

148. Kulkarni VM, Bodas D, Dhoble D, Ghormade V, Paknikar K. Radio-frequency triggered heating and drug release using doxorubicin-loaded LSMO nanoparticles for bimodal treatment of breast cancer. Colloids Surf B Biointerfaces. 2016; 145: 878-890. 
149. Wang L, et al. Radiofrequency-triggered tumor-targeting delivery system for theranostics application. ACS Appl Mater Interfaces. 2015; 7(10): 5736-47.

150. Somasundaram $\mathrm{VH}$, et al. Biodegradable Radiofrequency Responsive Nanoparticles for Augmented Thermal Ablation Combined with Triggered Drug Release in Liver Tumors. ACS Biomater Sci Eng. 2016; 2(5): 768-779.

151. Talekar M, et al. Translational Nano-Medicines: Targeted Therapeutic Delivery for Cancer and Inflammatory Diseases. AAPS Journal. 2015; 17(4): 813-827.

152. Masson JF, et al. Will nanobiosensors change therapeutic drug monitoring? The case of Methotrexate. Nanomedicine (Lond.). 2015; 10(4): 521-524.

153. Kijanka M, et al. Nanobody-based cancer therapy of solid tumors. Nanomedicine (Lond.). 2015; 10(1): 161-174.

154. Skotland T, et al. Development of nanoparticles for cancer use. Nanomedicine. 2014; 9(9): 1295-1299.

155. Muthu MS, et al. Nanotheranostics: advanced nanomedicine for the integration of diagnosis and therapy. Nanomedicine. 2014; 9(9): 1277-1280.

156. Bhojani MS, et al. Targeted Imaging and Therapy of Brain Cancer using Theranostic Nanoparticles. Mol Pharm. 2010; 7(6): 1921-1929.

157. Ng KK, Lovell JF, Zheng G. Lipoprotein-Inspired Nanoparticles for Cancer Theranostics. Accounts of Chemical Research. 2011; 44(10): 1105-1113.

158. Choi KY, et al. Theranostic nanoplatforms for simultaneous cancer imaging and therapy: current approaches and future perspectives. Nanoscale. 2012; $4(2): 330-342$

159. Fernandez-Fernandez A, et al. Theranostic applications of nanomaterials in cancer: Drug delivery, image-guided therapy and multifunctional platforms. Appl Biochem Biotechnol. 2011; 165(7-8): 1628-1651.

160. Sumer B, et al. Theranostic nanomedicine for cancer. Nanomedicine. 2008; 3(2): 137-140.

161. Luk BT, et al. Lipid- and Polymer-Based Nanostructures for Cancer Theranostics. Theranostics. 2012; 2(12):1117-1126

162. Kelkar SS, et al. Theranostics: Combining Imaging and Therapy. Bioconjugate Chem. 2011; 22: 1879-1903.

163. Benyettou F, Lalatonne Y, Chebbi I, et al. A multimodal magnetic resonance imaging nanoplatform for cancer theranostics. Phys Chem Phys. 2011; 13: 10020-10027.

164. Chen $\mathrm{W}, \mathrm{Xu} \mathrm{N}, \mathrm{Xu} \mathrm{L}$, et al. Multifunctional magnetoplasmonic nanoparticle assemblies for cancer therapy and diagnostics (theranostics). Macromol Rapid Commun. 2010; 31: 228-236.

165. Cole AJ, Yang VC, David AE. Cancer theranostics: the rise of targeted magnetic nanoparticles. Trends Biotechnol. 2011; 29: 323-332.

166. Lapotko D. Plasmonic Nanobubbles as Tunable Cellular Probes for Cancer Theranostics. Cancers (Basel). 2011; 3: 802-840.

167. Liao MY, Lai PS, Yu HP, et al. Innovative ligand-assisted synthesis of NIR-activated iron oxide for cancer theranostics. Chem Commun (Camb). 2012; 48: 5319-5321.

168. Povoski SP, Hatzaras IS, Mojzisik CM, Martin EW. Oncologic theranostics: recognition of this concept in antigen-directed cancer therapy for colorectal cancer with anti-TAG-72 monoclonal antibodies. Expert Rev Mol Diagn. 2011; 11: $667-670$.

169. Tong R, Hemmati HD, Langer R, Kohane DS. Photoswitchable nanoparticles for triggered tissue penetration and drug delivery. J Am Chem Soc. 2012; 134 : 8848-8855.

170. Harmsen S, et al. Surface-enhanced resonance Raman scattering nanostars for high-precision cancer imaging. Science Trans Med. 2015; 7(271).

171. Ahrens ET, Bulte JWM. Tracking immune cells in vivo using magnetic resonance imaging. Nat Rev Immunol. 2013; 13: 755-763.

172. Kron T. New developments in image guidance for radiotherapy. Cancer Forum. 2012; 36(2).

173. Baun A, Hansen SF. Environmental challenges for nanomedicine. Nanomedicine (Lond). 2008; 3: 605-608.

174. Bellare JR. Nanotechnology and nanomedicine for healthcare: challenges in translating innovations from bench to bedside. J Biomed Nanotechnol. 2011; 7: 36-37.

175. Kranz C, Eaton DC, Mizaikoff B. Analytical challenges in nanomedicine. Anal Bioanal Chem. 2011; 399: 2309-2311.

176. Lehr CM. Biological barriers and nanomedicine--timely challenges in advanced drug delivery research. Eur J Pharm Biopharm. 2009; 72: 287-288.

177. Master A, Livingston M, Sen Gupta A. Photodynamic nanomedicine in the treatment of solid tumors: Perspectives and challenges. J Control Release. 2013; 168: 88-102.

178. Pautler M, Brenner S. Nanomedicine: promises and challenges for the future of public health. Int J Nanomedicine. 2010; 5: 803-809.

179. Sanhai WR, Sakamoto JH, Canady R, Ferrari M. Seven challenges for nanomedicine. Nat Nanotechnol. 2008; 3: 242-244.

180. Thierry B, Textor M. Nanomedicine in focus: opportunities and challenges ahead. Biointerphases. 2012; 7: 19.

181. Ventola CL. The nanomedicine revolution: part 3: regulatory and safety challenges. P T. 2012; 37: 631-639.

182. Chen Q, Ke H, Dai Z, Liu Z. Nanoscale theranostics for physical stimulus responsive cancer therapies. Biomaterials. 2015; 73: 214-230.

183. Bansal A, Zhang Y. Photocontrolled Nanoparticle Delivery Systems for Biomedical Applications. Acc Chem Res. 2014; 47: 3052-3060.
184. Duncan R, Richardson SC. Endocytosis and intracellular trafficking as gateways for nanomedicine delivery: opportunities and challenges. Mol Pharm. 2012; 9: 2380-2402.

185. Feijen J, Hennink WE, Zhong Z. From innovative polymers to advanced nanomedicine: key challenges, recent progress and future perspectives: the second Symposium on Innovative Polymers for Controlled Delivery Suzhou, China, 11-14 September 2012. Nanomedicine (Lond.). 2013; 8: 177-180.

186. Khandare J, Calderon M, Dagia NM, Haag R. Multifunctional dendritic polymers in nanomedicine: opportunities and challenges. Chem Soc Rev. 2012; 41: $2824-2848$

187. King NM. Nanomedicine first-in-human research: challenges for informed consent. J Law Med Ethics. 2012; 40: 823-830.

188. Philippi C, Loretz B, Schaefer UF, Lehr CM. Telomerase as an emerging target to fight cancer--opportunities and challenges for nanomedicine. J Control Release. 2010; 146: 228-240.

189. Prabhakar U, Maeda H, Jain RK, et al. Challenges and Key Considerations of the Enhanced Permeability and Retention Effect for Nanomedicine Drug Delivery in Oncology. Cancer Res. 2013.

190. Tao L, Hu W, Liu Y, et al. Shape-specific polymeric nanomedicine: emerging opportunities and challenges. Exp Biol Med (Maywood). 2011; 236: 20-29.

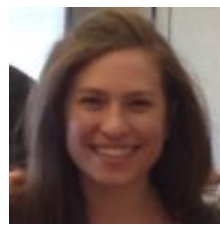

\section{Author Biography}

Alexandra Sneider obtained her bachelor's degree in Chemical Engineering from the University of Massachusetts Lowell in 2016, and has a previous BA in Anthropology and Archaeology from Harvard University in 2011. She will be starting her $\mathrm{PhD}$ in Chemical and Biomolecular Engineering at Johns Hopkins University in 2016. Alexandra was awarded a National Science Foundation Graduate Research Fellowship in 2016; American Institute of Chemists, Chemical Engineering Student Award 2016; American Institute of Chemical Engineers, Outstanding Chemical Engineering Student Award 2015; American Chemical Society, Division of Colloids 250 th Conference Travel Award 2015; and Materials Research Society, First Place Best Poster Winner

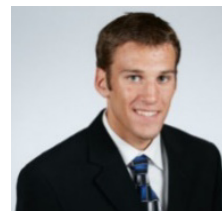
Boston 2014.

Derek VanDyke is currently a junior in the Department of Chemical Engineering at the University of Massachusetts Lowell. $\mathrm{He}$ has presented his research findings on silica-gold core-shells at the 250 th American Chemical Society National Meeting for which he was awarded the American Chemical Society Travel Award, the 90 th American Chemical Society Colloids Meeting, and will present at the 2016 American Institute of

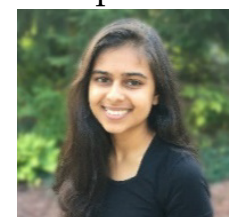
Chemical Engineers Annual Meeting.

Shailee Paliwal is a sophomore in the Department of Chemical Engineering at University of Massachusetts Lowell. She was awarded the Commonwealth Scholarship and is a member of the University of Massachusetts Lowell Honors College. She was also awarded the Co-Op Scholar Award during which she worked on gold and silica nanoparticles for theranostic applications. 


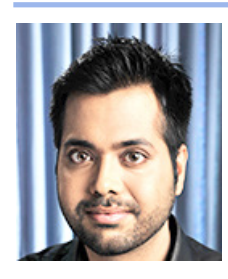

Dr. Prakash Rai is an assistant professor at the University of Massachusetts, Lowell in the department of chemical engineering. $\mathrm{He}$ received his BS in chemical engineering from the University of Mumbai, India in 2003. He obtained his MS and PhD degrees in chemical and biological engineering from Rensselaer Polytechnic Institute, Troy, NY in May and December of 2007 respectively. He is the author of more than 20 journal papers and one book chapter. His current research interests include nanotechnology, photodynamic therapy, fluorescence-based contrast agents, optical imaging, image-guided drug delivery and theranostics. He is a member of the American Institute of Chemical Engineers, American Physical Society, American Chemical Society, and the American Association of Cancer Research. 